\title{
AN INFRARED/X-RAY SURVEY FOR NEW MEMBERS OF THE TAURUS STAR-FORMING REGION*
}

\author{
K. L. Luhman ${ }^{1,4}$, E. E. MamajeK ${ }^{2}$, P. R. Allen ${ }^{1}$, and K. L. Cruz ${ }^{3,5}$ \\ ${ }^{1}$ Department of Astronomy and Astrophysics, The Pennsylvania State University, University Park, PA 16802, USA; kluhman@astro.psu.edu \\ ${ }^{2}$ Department of Physics and Astronomy, The University of Rochester, Rochester, NY 14627, USA \\ ${ }^{3}$ Astronomy Department, California Institute of Technology, Pasadena, CA 91125, USA \\ Received 2009 May 20; accepted 2009 July 31; published 2009 August 27
}

\begin{abstract}
We present the results of a search for new members of the Taurus star-forming region using data from the Spitzer Space Telescope and the XMM-Newton Observatory. We have obtained optical and near-infrared spectra of 44 sources that exhibit red Spitzer colors that are indicative of stars with circumstellar disks and 51 candidate young stars that were identified by Scelsi and coworkers using XMM-Newton. We also performed spectroscopy on four possible companions to members of Taurus that were reported by Kraus and Hillenbrand. Through these spectra, we have demonstrated the youth and membership of 41 sources, 10 of which were independently confirmed as young stars by Scelsi and coworkers. Five of the new Taurus members are likely to be brown dwarfs based on their late spectral types (>M6). One of the brown dwarfs has a spectral type of L0, making it the first known L-type member of Taurus and the least massive known member of the region $\left(M \sim 4-7 M_{\mathrm{Jup}}\right)$. Another brown dwarf exhibits a flat infrared spectral energy distribution, which indicates that it could be in the protostellar class I stage (star+disk+envelope). Upon inspection of archival images from various observatories, we find that one of the new young stars has a large edge-on disk $(r=2$ ".5 $=350 \mathrm{AU})$. The scattered light from this disk has undergone significant variability on a timescale of days in optical images from the Canada-France-Hawaii Telescope. Using the updated census of Taurus, we have measured the initial mass function for the fields observed by XMM-Newton. The resulting mass function is similar to previous ones that we have reported for Taurus, showing a surplus of stars at spectral types of K7-M1 (0.6$\left.0.8 M_{\odot}\right)$ relative to other nearby star-forming regions, such as IC 348, Chamaeleon I, and the Orion Nebula Cluster.
\end{abstract}

Key words: accretion, accretion disks - planetary systems: protoplanetary disks - stars: formation - stars: low-mass, brown dwarfs - stars: pre-main sequence

\section{INTRODUCTION}

The Taurus complex of dark clouds is one of the best sites for studying the formation of stars in a quiescent, relatively isolated environment. It is among the nearest star-forming regions $(d=$ $140 \mathrm{pc})$ and exhibits a very low stellar density $\left(n \sim 1-10 \mathrm{pc}^{-3}\right)$. Although the individual clouds are sparsely populated, the cloud complex as a whole contains more than 300 known members. Working toward a complete census of Taurus is important for the identification of rare objects (e.g., edge-on disks, transitional disks, protostars) as well as the statistical characterization of the stellar population (e.g., disk fraction, initial mass function (IMF), spatial distribution). A variety of methods have been employed in surveys for new members of Taurus (Kenyon et al. 2008). Two of these techniques, mid-infrared (IR) imaging and $\mathrm{X}$-ray imaging, are highly complementary. Mid-IR observations can identify stars that have circumstellar disks and can penetrate the high levels of extinction that surround stars at the earliest evolutionary stages while X-ray data can uncover the diskless members of a young stellar population.

Because of their excellent sensitivities and large fields of view, the Spitzer Space Telescope (Werner et al. 2004) and the XMM-Newton Observatory (Jansen et al. 2001) are the best available telescopes for wide-field imaging surveys at mid-IR

\footnotetext{
* Based on observations performed with the Hobby-Eberly Telescope, the Magellan Telescopes at Las Campanas Observatory, the NASA Infrared Telescope Facility, Gemini Observatory, the Spitzer Space Telescope, the XMM-Newton Observatory, and the Canada-France-Hawaii Telescope.

4 Visiting Astronomer at the Infrared Telescope Facility, which is operated by the University of Hawaii under Cooperative Agreement No. NCC 5-538 with the National Aeronautics and Space Administration (NASA), Office of Space Science, Planetary Astronomy Program.

5 Spitzer Fellow
}

and X-ray wavelengths, respectively. The unique capabilities of these facilities have been applied to the Taurus star-forming region through the Taurus Spitzer Legacy Survey (D. Padgett 2009, in preparation) and the XMM-Newton Extended Survey of the Taurus Molecular Cloud (XEST; Güdel et al. 2007). Luhman et al. (2006, 2009b) and Scelsi et al. (2007, 2008) have used the data from these surveys to search for new members of Taurus. We have continued those efforts by performing spectroscopy on IR sources that we have identified in the Spitzer images and $\mathrm{X}$-ray sources that were reported by Scelsi et al. (2007). In this paper, we describe the selection of these candidate members of Taurus (Section 2) and measure their spectral types with optical and IR spectra (Section 3). We then characterize the stellar parameters of the new members and discuss other notable properties of these objects (Section 4). Finally, we use our updated census of the stellar population in Taurus to measure the IMF within the fields observed by XEST (Section 5).

\section{SELECTION OF CANDIDATE MEMBERS OF TAURUS}

For the IR selection of candidate members of Taurus, we used images at 3.6, 4.5, 5.8, and $8.0 \mu \mathrm{m}$ obtained with Spitzer's Infrared Array Camera (IRAC; Fazio et al. 2004) and images at $24 \mu \mathrm{m}$ obtained with the Multiband Imaging Photometer for Spitzer (MIPS; Rieke et al. 2004). We considered all observations of this kind that have been performed in Taurus, many of which were collected through the Spitzer Legacy program of D. Padgett. These images of Taurus encompass a total area of $46 \mathrm{deg}^{2}$. The characteristics of the individual IRAC and MIPS observations are summarized by Luhman et al. (2009a), who present a compilation of 3.6-24 $\mu \mathrm{m}$ Spitzer photometry for all known members of Taurus. To identify 
candidate members of Taurus, we searched the Spitzer images for stars exhibiting red IRAC colors that are indicative of excess emission from circumstellar disks and envelopes. The reduction and analysis methods were the same as those employed in our previous surveys of star-forming regions (Luhman \& Muench 2008, references therein). In addition to sources with IRAC excesses, we also inspected the data for stars with excess emission at $24 \mu \mathrm{m}$ but not in the IRAC bands, which is a signature of a disk with an inner hole. We selected 44 of the resulting candidates for follow-up spectroscopy to determine whether they are members of Taurus. Two of these sources, FU Tau A and B, were reported in a separate study (Luhman et al. 2009 b). Because the secondary is too close to the primary for the measurement of IRAC photometry through our automated procedures, it was not identified as a candidate based on its IRAC colors. Instead, FU Tau B was selected for spectroscopy because of its close proximity to FU Tau A. Nevertheless, we count the former among the IR candidates for the purposes of this study. Through the spectroscopic observations described in the next section, we find that 24 of the 44 candidates are members of Taurus. One of these new members was detected by XMM-Newton (XEST 26-071) but was not recognized as a candidate member with those data (Scelsi et al. 2007).

We have included in our spectroscopic sample candidate members of Taurus that have been found through X-ray observations by the XMM-Newton Observatory. The XEST program (Güdel et al. 2007) obtained images of 19 fields in Taurus with $X M M-N e w t o n$ and utilized archival data for seven additional fields (one of which was observed twice). The boundaries of the XEST images are indicated on the map of the Taurus cloud complex in Figure 1. These fields are primarily located in the densest stellar aggregates and encompass a total area of $5 \mathrm{deg}^{2}$. Using these data, Scelsi et al. (2007) identified 57 possible members of Taurus. One of these candidates, XEST 13-010, was reported as a member by Luhman et al. (2006) while another candidate, XEST 06-045, is a galaxy according to images from the Digitized Sky Survey (DSS). We excluded from consideration four candidates that are far from the Taurus clouds ( $\alpha<4 \mathrm{hr})$. We selected the remaining 51 candidates for spectroscopy, 16 of which are classified as members of Taurus in the next section.

In addition to the IR and X-ray candidates, we have performed spectroscopy on four possible companions to known members of Taurus from Kraus \& Hillenbrand (2007), one of which is classified as a Taurus member through our spectroscopy. We also observed a previously known but widely overlooked member, LH 0429+17 (Reid \& Hawley 1999), so that we could measure its spectral type with the same classification methods that we have applied to the other late-type members of Taurus.

\section{SPECTROSCOPY OF CANDIDATES}

\subsection{Spectral Classification}

We performed optical and near-IR spectroscopy on the 100 targets selected in the previous section using a variety of instruments and telescopes. The dates, telescopes, and instrument configurations for these observations are summarized in Table 1. We examined these data for signatures of youth that indicate membership in Taurus, as done in our previous studies of this kind (e.g., Luhman 2004a). In addition, we considered other diagnostics of membership when available, such as proper motions (Luhman et al. 2009b; see the Appendix). Through this analysis, we classified 42 of the 100 targets in our spectroscopic sample as members of Taurus. The evidence of youth and membership for these sources is compiled in Table 2. The 58 nonmembers are listed in Table 3.

Most of the members of Taurus in our sample exhibit late-type ( $>\mathrm{M} 0)$ features in their spectra $\left(\mathrm{H}_{2} \mathrm{O}, \mathrm{TiO}, \mathrm{VO}\right)$. To measure spectral types for these sources, we have compared their spectra to previous data that we have collected for known members of Taurus and other star-forming regions (Luhman 2004a, 2004c, 2006), which were originally classified at optical wavelengths through comparison to averages of dwarfs and giants (Luhman 1999). One new member, 2MASS J04373705+2331080 (hereafter $2 \mathrm{M} 0437+2331)$, is later than all previously known members of Taurus ( $\leqslant$ M9.5). We classified this object through a comparison to standard L dwarfs (Kirkpatrick et al. 1999) and young L dwarfs in the field ( $\tau \sim 10-100 \mathrm{Myr}$, Kirkpatrick et al. 2006; Cruz et al. 2009), arriving at a spectral type of L0 (see Figure 2). One new member with a K-type spectrum, HQ Tau, was classified with dwarf standards (Allen \& Strom 1995). We could not measure a spectral type for one of the new members, 2MASS J04293209+2430597, because photospheric features are not detected in its spectrum. Our spectral classifications for the Taurus members are provided in Table 2. The spectra are shown in order of spectral type in Figures 3-5. The highest resolution data are presented in Figure 6 for the wavelength range encompassing $\mathrm{H} \alpha$ and $\mathrm{Li}$ I. The equivalent widths of $\mathrm{Li}$ I from these spectra are given in Table 4 . The positions of the 41 new members (excluding LH 0429+17) are plotted on the map of Taurus in Figure 1.

Among the nonmembers, we classified field stars with standard dwarfs and giants (Henry et al. 1994; Kirkpatrick et al. 1991, 1997; Cushing et al. 2005) and we identified galaxies based on the presence of redshifted emission lines. The classifications of nonmembers are found in Table 3.

Scelsi et al. (2008) obtained optical spectra of 25 of the candidate members of Taurus from the XEST program (Scelsi et al. 2007). They classified 10 candidates as young stars and 12 candidates as nonmembers. We observed 20 of these 22 sources; our membership classifications agree with those of Scelsi et al. (2008) in all cases. The membership of the remaining three candidates was uncertain based on the data from Scelsi et al. (2008). We find that two of these objects are foreground dwarfs (XEST 08-014, XEST 15-034) while the other candidate is a Taurus member (XEST 20-071). Our spectral types are systematically later than those from Scelsi et al. (2008) by a few subclasses for sources in common between the two studies.

\subsection{Comments on Individual Sources}

Several of our targets have displayed evidence of membership in Taurus in previous studies, although they lacked spectroscopic classifications. The previous observations of one of these objects, FU Tau, are described in detail by Luhman et al. (2009b). Torres et al. (1995) identified 2MASS J04455134+1555367 as a possible young star based on mid-IR photometry from the Infrared Astronomical Satellite (IRAS). ${ }^{6}$ They detected emission in $\mathrm{H} \alpha$ and $\mathrm{He}$ I and absorption in $\mathrm{Li}$ I through follow-up spectroscopy. Jones \& Herbig (1979) found that the proper motion of HQ Tau is consistent with membership in Taurus. Mid-IR excess emission also was detected toward this star in photometry from IRAS (Harris et al. 1988) and a spectrum from Spitzer

\footnotetext{
6 2MASS J04455134+1555367 and its 20" companion HD 30171 were unresolved in the IRAS data. Images at higher resolution from Spitzer demonstrate that 2MASS J04455134+1555367 was the source of the IRAS emission (Luhman et al. 2009a).
} 


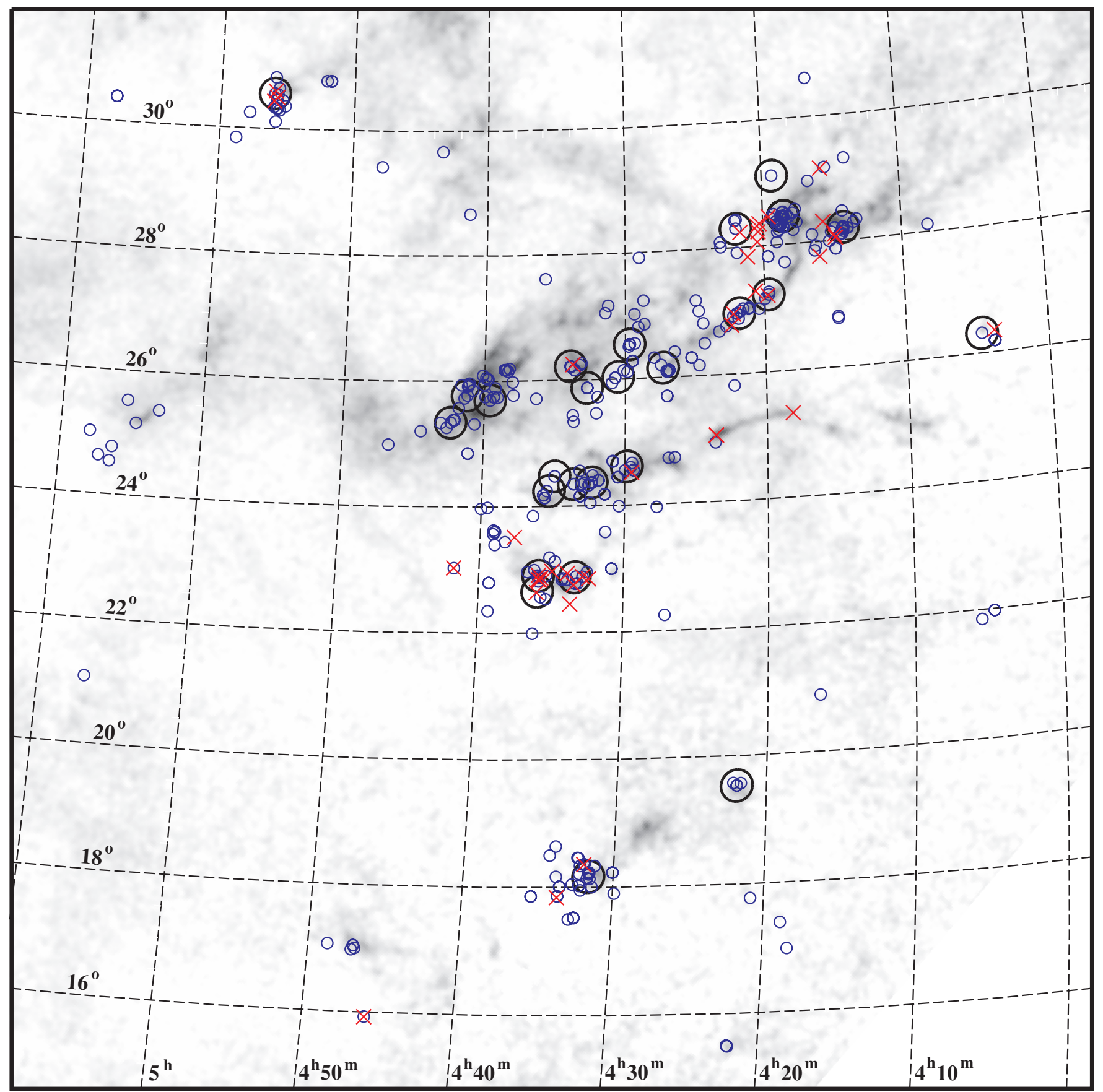

Figure 1. Spatial distribution of previously known members of the Taurus star-forming region (small circles) and new objects that we have confirmed as members through spectroscopy (crosses). The latter were identified as possible members with the XMM-Newton Observatory (Scelsi et al. 2007), the Spitzer Space Telescope (this work), and a companion survey with 2MASS (Kraus \& Hillenbrand 2007). The fields imaged with XMM-Newton are indicated (large circles). The dark clouds in Taurus are displayed with a map of extinction (gray scale; Dobashi et al. 2005).

(Furlan et al. 2006). Kenyon et al. (1994) identified V409 Tau and IRAS $04125+2902$ as candidate members of Taurus based on IRAS data. They classified the latter as a galaxy through near-IR images or optical spectroscopy, but it is an M-type star according to our spectroscopy (see Figure 3).

We now discuss the stars in our sample that have uncertain classifications. Our spectrum of 2MASS J04293209+2430597 does not show any absorption or emission lines that would demonstrate that it is a young star. However, its very red, featureless spectrum and mid-IR excess emission are consistent with a protostar. Given its close proximity to a dark cloud and other known members of Taurus, we tentatively classify it as a member. Based on its strong $\mathrm{H} \alpha$ emission $\left[W_{\lambda}(\mathrm{H} \alpha)=58 \pm\right.$ $1 \AA]$ and mid-IR excess emission, 2MASS J04124858+2749563 is clearly a young star. However, it is much fainter than members of Taurus near its spectral type and its proper motion is inconsistent with membership (Monet et al. 2003; Zacharias et al. 2004b; see the Appendix). Therefore, 2MASS J04124858+2749563 is probably a background star (albeit a young one). Our IR spectrum of 2MASS J04345973+2807017 is better matched by dwarf standards, but it does not have a sufficient signal-to-noise ratio to rule out youth. This star is anomalously faint for a Taurus member and is far from known members of the region, which suggest that it is a background star. Stars with edge-on 
Table 1

Observing Log

\begin{tabular}{|c|c|c|c|c|c|}
\hline Night & Date & Telescope + Instrument & Disperser & $\begin{array}{c}\lambda \\
(\mu \mathrm{m}) \\
\end{array}$ & $\lambda / \Delta \lambda$ \\
\hline 1 & 2006 Sep 25 & HET + LRS & G3 grism & $0.63-0.91$ & 1100 \\
\hline 2 & 2006 Oct 2 & HET + LRS & G3 grism & $0.63-0.91$ & 1100 \\
\hline 3 & 2006 Oct 3 & HET + LRS & G3 grism & $0.63-0.91$ & 1100 \\
\hline 4 & 2006 Oct 19 & HET + LRS & G3 grism & $0.63-0.91$ & 1100 \\
\hline 5 & 2006 Oct 20 & HET + LRS & G3 grism & $0.63-0.91$ & 1100 \\
\hline 6 & 2006 Oct 22 & HET + LRS & G3 grism & $0.63-0.91$ & 1100 \\
\hline 7 & 2006 Nov 17 & HET + LRS & G3 grism & $0.63-0.91$ & 1100 \\
\hline 8 & 2006 Nov 26 & HET + LRS & G3 grism & $0.63-0.91$ & 1100 \\
\hline 9 & 2007 Feb 19 & IRTF + SpeX & Prism & $0.8-2.5$ & 100 \\
\hline 10 & 2007 Aug 13 & HET + MRS & $791 / \mathrm{mm}$ grating & $0.45-0.9$ & 11000 \\
\hline 11 & 2007 Aug 28 & HET + LRS & G3 grism & $0.63-0.91$ & 1100 \\
\hline 12 & 2007 Sep 15 & HET + LRS & G3 grism & $0.63-0.91$ & 1100 \\
\hline 13 & 2007 Sep 26 & HET + MRS & $791 / \mathrm{mm}$ grating & $0.45-0.9$ & 11000 \\
\hline 14 & 2007 Oct 3 & HET + MRS & $791 / \mathrm{mm}$ grating & $0.45-0.9$ & 11000 \\
\hline 15 & 2007 Oct 10 & HET + MRS & $791 / \mathrm{mm}$ grating & $0.45-0.9$ & 11000 \\
\hline 16 & 2007 Oct 20 & HET + MRS & $791 / \mathrm{mm}$ grating & $0.45-0.9$ & 11000 \\
\hline 17 & 2007 Oct 22 & HET + LRS & G3 grism & $0.63-0.91$ & 1100 \\
\hline 18 & 2007 Oct 23 & HET + LRS & G3 grism & $0.63-0.91$ & 1100 \\
\hline 19 & 2007 Oct 24 & HET + LRS & G3 grism & $0.63-0.91$ & 1100 \\
\hline 20 & 2007 Oct 25 & HET + LRS & G3 grism & $0.63-0.91$ & 1100 \\
\hline 21 & 2007 Oct 26 & HET + LRS & G3 grism & $0.63-0.91$ & 1100 \\
\hline 22 & 2007 Nov 1 & HET + LRS & G3 grism & $0.63-0.91$ & 1100 \\
\hline 23 & 2007 Nov 3 & HET + LRS & G3 grism & $0.63-0.91$ & 1100 \\
\hline 24 & 2007 Nov 3 & HET + MRS & $791 / \mathrm{mm}$ grating & $0.45-0.9$ & 11000 \\
\hline 25 & 2007 Nov 4 & HET + LRS & G3 grism & $0.63-0.91$ & 1100 \\
\hline 26 & 2007 Nov 13 & HET + LRS & G3 grism & $0.63-0.91$ & 1100 \\
\hline 27 & 2007 Nov 15 & HET + LRS & G3 grism & $0.63-0.91$ & 1100 \\
\hline 28 & 2007 Nov 28 & HET + LRS & G3 grism & $0.63-0.91$ & 1100 \\
\hline 29 & 2007 Dec 3 & HET + LRS & G3 grism & $0.63-0.91$ & 1100 \\
\hline 30 & 2007 Dec 3 & IRTF + SpeX & Prism & $0.8-2.5$ & 100 \\
\hline 31 & 2007 Dec 14 & HET + LRS & G1 grism & $0.57-1.03$ & 750 \\
\hline 32 & 2007 Dec 17 & Magellan II + LDSS-3 & VPH all grism & $0.58-1.1$ & 750 \\
\hline 33 & 2007 Dec 18 & Magellan II + LDSS-3 & VPH red grism & $0.6-1$ & 1400 \\
\hline 34 & 2007 Dec 20 & HET + MRS & $791 / \mathrm{mm}$ grating & $0.45-0.9$ & 11000 \\
\hline 35 & 2007 Dec 23 & HET + LRS & G1 grism & $0.57-1.03$ & 750 \\
\hline 36 & 2008 Jan 6 & HET + LRS & G1 grism & $0.57-1.03$ & 750 \\
\hline 37 & 2008 Jan 10 & HET + LRS & G1 grism & $0.57-1.03$ & 750 \\
\hline 38 & 2008 Jan 13 & HET + MRS & $791 / \mathrm{mm}$ grating & $0.45-0.9$ & 11000 \\
\hline 39 & 2008 Jan 13 & HET + LRS & G1 grism & $0.57-1.03$ & 750 \\
\hline 40 & 2008 Jan 15 & HET + MRS & 79 1/mm grating & $0.45-0.9$ & 11000 \\
\hline 41 & 2008 Feb 17 & HET + MRS & 79 1/mm grating & $0.45-0.9$ & 11000 \\
\hline 42 & 2008 Sep 1 & Gemini + GMOS & $4001 / \mathrm{mm}$ grating & $0.56-1$ & 1500 \\
\hline 43 & 2008 Sep 13 & HET + LRS & G3 grism & $0.63-0.91$ & 1100 \\
\hline 44 & 2008 Sep 30 & Gemini + NIRI & $\mathrm{f} / 6 \mathrm{~K}$ grism & $1.9-2.5$ & 500 \\
\hline 45 & 2008 Oct 2 & HET + LRS & G3 grism & $0.63-0.91$ & 1100 \\
\hline 46 & 2008 Oct 10 & Gemini + NIRI & $\mathrm{f} / 6 \mathrm{~K}$ grism & $1.9-2.5$ & 500 \\
\hline 47 & 2008 Oct 23 & HET + LRS & G3 grism & $0.63-0.91$ & 1100 \\
\hline 48 & 2008 Nov 2 & IRTF + SpeX & Prism & $0.8-2.5$ & 100 \\
\hline 49 & 2009 Feb 12 & IRTF + SpeX & Prism & $0.8-2.5$ & 100 \\
\hline 50 & 2009 Feb 14 & HET + LRS & G1 grism & $0.57-1.03$ & 750 \\
\hline
\end{tabular}

Notes. The Gemini data were obtained through program GN-2008B-Q-21. The data from SpeX (Rayner et al. 2003) were reduced with the Spextool package (Cushing et al. 2004) and corrected for telluric absorption (Vacca et al. 2003).

disks also appear very faint for their spectral types. 2MASS J04345973+2807017 does appear to have excess emission at $8 \mu \mathrm{m}$ that indicates the presence of a disk, but its large distance from known members of Taurus tends to support a classification as a background star. Finally, the proper motion of the A-type star 2MASS J04180338+2440096 is inconsistent with membership in Taurus (Høg et al. 2000; Zacharias et al. 2004b; Röser et al. 2008; see the Appendix), but it exhibits mid-IR excesses of 0.2 and 3 mag at 8 and $24 \mu \mathrm{m}$, respectively. It is probably a field star with a debris disk.

\section{PROPERTIES OF NEW MEMBERS}

\subsection{Temperatures and Luminosities}

We have estimated the effective temperatures and bolometric luminosities of the Taurus members in our spectroscopic 
Table 2

Members of Taurus in Spectroscopic Sample

\begin{tabular}{|c|c|c|c|c|c|c|c|c|c|}
\hline $2 \mathrm{MASS}^{\mathrm{a}}$ & Other Names & $\begin{array}{l}\text { Spectral } \\
\text { Type }^{\mathrm{b}}\end{array}$ & $\begin{array}{c}T_{\text {eff }}^{\mathrm{c}} \\
(\mathrm{K})\end{array}$ & $A_{J}$ & $\begin{array}{l}L_{\text {bol }} \\
\left(L_{\odot}\right) \\
\end{array}$ & $\begin{array}{l}\text { Membership } \\
\text { Evidence }^{\mathrm{d}}\end{array}$ & $\begin{array}{c}W_{\lambda}(\mathrm{H} \alpha) \\
(\AA)\end{array}$ & $\begin{array}{c}\text { Basis of } \\
\text { Selection }^{\mathrm{e}} \\
\end{array}$ & Night \\
\hline J04034997+2620382 & XEST 06-006 & M5.25 & 3091 & 0 & 0.012 & $\mathrm{NaK}, \mu$ & $9 \pm 0.5$ & X-ray & 11 \\
\hline J04144739+2803055 & XEST 20-066 & M5.25 & 3091 & 0 & 0.12 & $\mathrm{NaK}, \mu$ & $8.5 \pm 0.5$ & X-ray & 21 \\
\hline J04145234+2805598 & XEST 20-071 & M3.25 & 3379 & 0.78 & 0.84 & $A_{V}, \mathrm{NaK}, \mu$ & $7.5 \pm 0.5$ & X-ray & 17 \\
\hline J04153916+2818586 & $\ldots$ & M3.75 & 3306 & 0.56 & 0.27 & ex, $\mathrm{NaK}, A_{V}$ & $14 \pm 1$ & IR & 7 \\
\hline J04154278+2909597 & IRAS $04125+2902$ & M1.25 & 3669 & 0.56 & 0.28 & $A_{V}, \mathrm{ex}, \mu$ & $2.3 \pm 0.3$ & IR & 45 \\
\hline J04155799+2746175 & $\ldots$ & M5.5 & 3058 & 0 & 0.049 & $\mathrm{NaK}, \mathrm{e}, \mathrm{ex}$ & $39 \pm 1$ & IR & 19 \\
\hline J04181078+2519574 & V409 Tau, IRAS 04151+2512 & M1.5 & 3632 & 1.3 & 0.53 & $\mathrm{Li}, \mathrm{ex}, \mathrm{e}, \mu$ & $10 \pm 1$ & IR & 14 \\
\hline J04193545+2827218 & FR Tau, Haro6-4, XEST 23-076, XEST 24-063 & M5.25 & 3091 & 0 & 0.10 & $\mathrm{e}, \mathrm{ex}, \mathrm{NaK}, \mu$ & $67 \pm 5$ & IR & 6 \\
\hline J04194657+2712552 & {$[\mathrm{GKH} 94] 41$} & $\mathrm{M} 7.5 \pm 1.5$ & 2795 & 7.6 & 0.24 & $A_{V}$, ex, $\mathrm{H}_{2} \mathrm{O}$ & & IR & 48 \\
\hline J04201611+2821325 & $\ldots$ & M6.5 & 2935 & 0 & 0.0072 & e, ex, $\mathrm{NaK}$ & $1.0 \pm 30$ & IR & 1 \\
\hline J04202144+2813491 & $\ldots$ & $\mathrm{M} 1 \pm 2$ & 3705 & $\ldots$ & $\ldots$ & $A_{V}, \mathrm{e}, \mathrm{ex}$ & $>100$ & IR & 50 \\
\hline J04202583+2819237 & IRAS $04173+2812$ & mid-M & $\cdots$ & $\cdots$ & $\cdots$ & e, ex & $\ldots$ & IR & 9 \\
\hline J04202606+2804089 & $\ldots$ & M3.5 & 3342 & 0 & 0.15 & ex, $\mathrm{NaK}$ & $5 \pm 0.3$ & IR & 4 \\
\hline $\mathrm{J} 04203918+2717317^{\mathrm{f}}$ & XEST 16-045 & M4.5 & 3198 & 0 & 0.16 & $\mathrm{NaK}, \mu$ & $8 \pm 0.5$ & $\mathrm{X}$-ray & 21 \\
\hline J04210934+2750368 & $\ldots$ & M5.25 & 3091 & 0 & 0.079 & ex, $\mathrm{NaK}$ & $20 \pm 2$ & IR & 6 \\
\hline J04214013+2814224 & XEST $21-026$ & M5.75 & 3024 & 0 & 0.040 & $\mathrm{NaK}, \mu$ & $7 \pm 0.5$ & X-ray & 21 \\
\hline $\mathrm{J} 04221568+2657060^{\mathrm{f}}$ & XEST $11-078$, IRAS $04192+2650$ & M1 & 3705 & 0.28 & $\ldots$ & $A_{V}, \mathrm{e}, \mathrm{ex}, \mu$ & $14 \pm 1$ & $\mathrm{X}$-ray & 21 \\
\hline J04222404+2646258 ${ }^{\mathrm{f}}$ & XEST $11-087$ & M4.75 & 3161 & 0.31 & 0.12 & $A_{V}, \mathrm{NaK}$ & $7.5 \pm 1$ & $\mathrm{X}$-ray & 20 \\
\hline J04233539+2503026 & FU Tau A & M7.25 & 2838 & 0.56 & 0.19 & $\mathrm{ex}, \mathrm{H}_{2} \mathrm{O}, \mathrm{NaK}, \mathrm{e}, \mu$ & $93 \pm 7$ & IR & 30,32 \\
\hline J04233573+2502596 & FU Tau B & M9.25 & 2350 & 0 & 0.0025 & $\mathrm{NaK}, \mathrm{ex}, \mathrm{e}$ & $\sim 70$ & IR & 33 \\
\hline J04293209+2430597g & $\ldots$ & $?$ & $\ldots$ & $\ldots$ & $\ldots$ & ex & $\ldots$ & IR & 9 \\
\hline J04315968+1821305 & LkHa 267 & $\mathrm{M} 1.5 \pm 0.5$ & 3632 & 1.4 & 0.14 & $A_{V}, \mathrm{e}, \mathrm{ex}$ & $\ldots$ & IR & 49 \\
\hline J04322415+2251083 & $\ldots$ & M4.5 & 3198 & 0.49 & 0.090 & $A_{V}, \mathrm{NaK}, \mathrm{ex}$ & $14 \pm 1$ & IR & 18 \\
\hline J04324938+2253082 & $\ldots$ & M4.25 & 3234 & 0.87 & 0.21 & $A_{V}$, ex, $\mathrm{NaK}$ & $18.5 \pm 1$ & IR & 22 \\
\hline J04325119+1730092 & LH 0429+17 & M8.25 & 2632 & 0 & 0.0033 & $\mathrm{H}_{2} \mathrm{O}, \mathrm{NaK}, \mu$ & $17 \pm 10$ & classify & 30,33 \\
\hline $\mathrm{J} 04332621+2245293$ & XEST 17-036 & M4 & 3270 & 1.1 & 0.14 & $\mathrm{NaK}, A_{V}$ & $6 \pm 1$ & $\mathrm{X}$-ray & 31 \\
\hline J04333905+2227207 & $\ldots$ & M1.75 & 3596 & 0.35 & $\ldots$ & $A_{V}, \mathrm{e}, \mathrm{ex}$ & $23 \pm 1$ & IR & 17 \\
\hline J04334171+1750402 & $\cdots$ & $\mathrm{M} 4 \pm 0.5$ & 3270 & 0.08 & 0.088 & $A_{V}$, ex, $\mathrm{H}_{2} \mathrm{O}$ & $\ldots$ & IR & 49 \\
\hline J04334465+2615005 & $\ldots$ & M4.75 & 3161 & 0.85 & 0.12 & $\mathrm{e}, \mathrm{ex}, \mathrm{NaK}, A_{V}$ & $55 \pm 7$ & IR & 3 \\
\hline$J 04335252+2256269^{\mathrm{f}}$ & XEST 17-059 & M5.75 & 3024 & 0 & 0.19 & $\mathrm{NaK}, \mu$ & $17 \pm 1$ & $\mathrm{X}$-ray & 21 \\
\hline $\mathrm{J} 04345693+2258358^{\mathrm{f}}$ & XEST 08-003 & M1.5 & 3632 & 0.54 & 0.34 & $\mathrm{Li}, \mu$ & $2 \pm 0.2$ & X-ray & 13 \\
\hline $\mathrm{J} 04354203+2252226^{\mathrm{f}}$ & XEST 08-033, XEST 09-023 & M4.75 & 3161 & 0.49 & 0.12 & $A_{V}, \mathrm{NaK}, \mu$ & $14 \pm 1$ & $\mathrm{X}$-ray & 20 \\
\hline J04354733+2250216 & $\begin{array}{c}\text { HQ Tau, IRAS 04327+2244, XEST 09-026, } \\
\text { XEST 08-037 }\end{array}$ & $\mathrm{K} 2 \pm 2$ & 4900 & 1.2 & 4.6 & $\mathrm{Li}, \mathrm{ex}, \mu$ & $2 \pm 0.4$ & IR & 10 \\
\hline$J 04355209+2255039^{f}$ & XEST $08-047$ & M4.5 & 3198 & 0.56 & 0.13 & $A_{V}, \mathrm{NaK}, \mu$ & $6 \pm 0.5$ & X-ray & 21 \\
\hline$J 04355286+2250585^{f}$ & XEST 08-049, XEST 09-033 & M4.25 & 3234 & 0.35 & 0.14 & $\mathrm{NaK}, \mathrm{e}, \mu$ & $25.5 \pm 1$ & X-ray & 27 \\
\hline$J 04355892+2238353^{\mathrm{f}}$ & XEST 09-042 & M0 & 3850 & 0.11 & 0.71 & $\mathrm{Li}, \mu$ & $1.4 \pm 0.1$ & X-ray & 16 \\
\hline J04373705+2331080 & $\ldots$ & $\mathrm{L} 0 \pm 0.5$ & 2200 & 0 & 0.0003 & $\mathrm{NaK}$ & $>50$ & IR & 42 \\
\hline J04414565+2301580 & $\ldots$ & $\mathrm{M} 4.5 \pm 0.5$ & 3198 & 0.11 & 0.14 & $\mathrm{H}_{2} \mathrm{O}$ & $\cdots$ & comp & 30 \\
\hline J04455134+1555367 & IRAS $04429+1550$ & $\mathrm{M} 2.5 \pm 0.5$ & 3488 & 0.42 & 0.32 & $\mathrm{Li}, A_{V}$, ex & $\ldots$ & IR & 49 \\
\hline J04554820+3030160 & XEST 26-052 & M4.5 & 3198 & 0 & 0.045 & $\mathrm{NaK}, \mu$ & $6 \pm 0.5$ & X-ray & 17 \\
\hline$J 04555605+3036209^{f}$ & JH433, XEST 26-062 & M4 & 3270 & 0.53 & 0.28 & $\mathrm{Li}, \mathrm{e}, \mathrm{ex}$ & $17 \pm 2$ & X-ray & 24 \\
\hline J04560118+3026348 & XEST 26-071 & $\mathrm{M} 3.5 \pm 0.5$ & 3342 & 0.39 & 0.11 & $A_{V}$, ex,$\mu$ & $\ldots$ & IR & 49 \\
\hline
\end{tabular}

Notes.

a 2MASS Point Source Catalog.

${ }^{\mathrm{b}}$ Uncertainties are \pm 0.25 subclass unless noted otherwise.

${ }^{c}$ Temperature scale from Luhman et al. (2003b).

d Membership in Taurus is indicated by $A_{V} \gtrsim 1$ and a position above the main sequence for the distance of Taurus (" $A_{V}$ "), strong emission lines ("e"), Na I and K I strengths intermediate between those of dwarfs and giants ("NaK"), strong Li absorption ("Li"), IR excess emission ("ex"), the shape of the gravity-sensitive steam bands (" $\mathrm{H}_{2} \mathrm{O}$ "), or a proper motion that is similar to that of the known members of Taurus (" $\mu$ ").

e Sources were selected for spectroscopy because they were candidate companions to known Taurus members ("comp”, Kraus \& Hillenbrand 2007) or they were identified as candidate members with XMM-Newton ("X-ray", Scelsi et al. 2007) or Spitzer ("IR", this work). Spectroscopy was also performed on a previously known member to obtain a new spectral classification ("classify", Reid \& Hawley 1999).

${ }^{\mathrm{f}}$ Independently confirmed as a member through spectroscopy by Scelsi et al. (2008).

${ }^{\mathrm{g}}$ Membership is uncertain because of the absence of spectroscopic evidence of youth.

sample so that we can place them on the Hertzsprung-Russell (H-R) diagram. During spectral classification, we measured extinctions from the slopes of most of our optical and near-IR spectra (Luhman 2004a, 2007). We could not use the MRS spectra for this purpose because they were obtained through fibers rather than slits aligned at the parallactic angle, making the data susceptible to differential refraction and the spectral slopes unreliable. For the Medium Resolution Spectrograph (MRS) targets, we computed extinctions from $J-H$ and $H-K_{s}$ in the manner described by Luhman (2004a). We estimated luminosities by combining the extinctions with $J$-band photometry from the Point Source Catalog of the Two Micron All Sky Survey (2MASS; Skrutskie et al. 2006), a distance of 140 pc (Wichmann et al. 1998; Loinard et al. 2005, 2007; Torres et al. 2007, 
Table 3

Nonmembers in Spectroscopic Sample

\begin{tabular}{|c|c|c|c|c|}
\hline $\mathrm{ID}^{\mathrm{a}}$ & Other Names & $\begin{array}{c}\text { Spectral } \\
\text { Type } \\
\end{array}$ & $\begin{array}{c}\text { Basis of } \\
\text { Selection }^{\mathrm{b}}\end{array}$ & Night \\
\hline 2MASS J04042449+2611119 & XEST 06-041 & $\mathrm{M} 2.5 \mathrm{~V}$ & X-ray & 20 \\
\hline 2MASS J04124858+2749563 & $\ldots$ & $\mathrm{K} 0-\mathrm{K} 4$ & IR & 19 \\
\hline 2MASS J04141588+2818181 & XEST 20-045 & $<\mathrm{M} 4$ & X-ray & 30 \\
\hline 2MASS J04144294+2821105 & XEST 20-063 & $<\mathrm{M} 4$ & $\mathrm{X}$-ray & 30 \\
\hline 2MASS J04164774+2408242 & $\ldots$ & $<\mathrm{M} 0$ & IR & 18 \\
\hline 2MASS J04170711+2408041 & $\ldots$ & Galaxy & IR & 19 \\
\hline 2MASS J04180338+2440096 & $\cdots$ & Early A & IR & 49 \\
\hline 2MASS J04180674+2904015 & $\cdots$ & $<\mathrm{M} 0$ & IR & 18 \\
\hline 2MASS J04182321+2519280 & $\ldots$ & Galaxy & IR & 17 \\
\hline 2MASS J04190125+2837101 & XEST $23-062$ & Giant & X-ray & 22 \\
\hline 2MASS J04190689+2826090 & XEST 23-065,XEST 24-057 & $<\mathrm{M} 4$ & X-ray & 30 \\
\hline 2MASS J04191612+2750481 & $\ldots$ & Galaxy & IR & 3 \\
\hline 2MASS J04214372+2647225 & XEST 11-035 & $<\mathrm{M} 0$ & X-ray & 16 \\
\hline 2MASS J04221295+2546598 & $\ldots$ & Galaxy & IR & 30 \\
\hline 2MASS J04221918+2348005 & $\ldots$ & Galaxy & IR & 12 \\
\hline 2MASS J04222559+2812332 & XEST 21-059 & $<\mathrm{M} 0$ & X-ray & 16 \\
\hline 2MASS J04222718+2659512 & XEST 11-088 & $\mathrm{M} 3.25 \mathrm{~V}$ & $\mathrm{X}$-ray & 23 \\
\hline 2MASS J04223441+2457186 & $\ldots$ & Galaxy & IR & 23 \\
\hline 2MASS J04224865+2823005 & XEST 21-073 & M4V & $\mathrm{X}$-ray & 26 \\
\hline 2MASS J04263497+2608161 & XEST 02-005 & Galaxy & $\mathrm{X}$-ray & 39 \\
\hline 2MASS J04275871+2611062 & XEST 02-069 & $<\mathrm{M} 0$ & $\mathrm{X}$-ray & 40 \\
\hline 2MASS J04285844+2436492 & XEST 13-002 & M0 & X-ray & 47 \\
\hline 2MASS J04292083+2742074 & IRAS $04262+2735$ & M5III & IR & 4 \\
\hline 2MASS J04292887+2616483c & $\ldots$ & Giant & comp & 48 \\
\hline 2MASS J04293623+2634238 & LP358-731,XEST 15-034 & $\mathrm{M} 4.25 \mathrm{~V}$ & X-ray & 22 \\
\hline 2MASS J04301702+2622264 & LP358-352,XEST 15-075 & M4.5V & X-ray & 29 \\
\hline 2MASS J04302526+2602566 & XEST $14-034$ & $\mathrm{M} 0.5 \mathrm{~V}$ & X-ray & 43 \\
\hline 2MASS J04302710+2807073 & IRAS $04273+2800$ & Galaxy & IR & 3 \\
\hline 2MASS J04304153+2430416 & XEST 13-036 & $<\mathrm{M} 0$ & $\mathrm{X}$-ray & 16 \\
\hline 2MASS J04314419+2813170 & $\ldots$ & Galaxy & IR & 8 \\
\hline 2MASS J04314634+2558404 & XEST 19-002 & Giant? & $\mathrm{X}$-ray & 17 \\
\hline 2MASS J04315860+1818408 & XEST 22-071 & $<\mathrm{M} 0$ & X-ray & 41 \\
\hline 2MASS J04322689+1818230 & XEST 22-111 & $\mathrm{M} 2.25 \mathrm{~V}$ & $\mathrm{X}$-ray & 20 \\
\hline 2MASS J04322946+1814002 & XEST $22-114$ & Galaxy & X-ray & 20 \\
\hline IRAC J04323521+2420213 & XEST 03-026,XEST 04-001 & Galaxy & IR & 44 \\
\hline 2MASS J04323605+2552225 & XEST $19-041$ & Giant & X-ray & 28 \\
\hline 2MASS J04323949+2427043 & XEST 03-028 & $<\mathrm{M} 4$ & X-ray & 30 \\
\hline 2MASS J04325921+2430403 & XEST 03-033,XEST 04-005 & Galaxy & X-ray & 28 \\
\hline 2MASS J04332491+2559262 & XEST $19-083$ & Giant & X-ray & 23 \\
\hline 2MASS J04333301+2252521 & XEST $17-043$ & $\mathrm{M} 4.5 \mathrm{~V}$ & X-ray & 36 \\
\hline 2MASS J04333746+2609550 & $\ldots$ & $<\mathrm{M} 4$ & comp & 30 \\
\hline 2MASS J04335562+2425016 & XEST 04-060 & $<\mathrm{M} 0$ & X-ray & 34 \\
\hline 2MASS J04341498+2826124 & $\ldots$ & Giant & IR & 5 \\
\hline 2MASS J04343322+2602403 & JH87,XEST 18-059 & $<\mathrm{M} 0$ & X-ray & 38 \\
\hline 2MASS J04345164+2404426 & XEST 12-012 & $\mathrm{M} 3.5 \mathrm{~V}$ & X-ray & 35 \\
\hline 2MASS J04345973+2807017 & $\ldots$ & M7V? & IR & 9 \\
\hline 2MASS J04351316+2259205 & EZ Tau,LP358-739,XEST 08-014 & $\mathrm{M} 4.75 \mathrm{~V}$ & X-ray & 22 \\
\hline 2MASS J04353651+2304590 & XEST 08-027 & $\mathrm{M} 3.25 \mathrm{~V}$ & $\mathrm{X}$-ray & 45 \\
\hline 2MASS J04354076+2411211 & $\ldots$ & $<\mathrm{M} 4$ & comp & 30 \\
\hline 2MASS J04400363+2553547 & [GKH94] 6,XEST 05-027 & Giant & X-ray & 19 \\
\hline 2MASS J04403912+2540024 & XEST 07-005 & $\mathrm{M} 4.5 \mathrm{~V}$ & X-ray & 37 \\
\hline IRAC J04412575+2543492 & XEST 07-032 & Galaxy & IR & 46 \\
\hline 2MASS J04415577+2302532 & $\ldots$ & Galaxy & IR & 30 \\
\hline 2MASS J04420376+2519533 & XEST 10-016 & Giant? & X-ray & 25 \\
\hline 2MASS J04455482+2408435 & IRAS $04428+2403$ & Galaxy & IR & 1 \\
\hline 2MASS J04455704+2440423 & $\ldots$ & Giant & IR & 2 \\
\hline 2MASS J04553844+3031465 & JH427,XEST 26-031 & $<\mathrm{M} 0$ & $\mathrm{X}$-ray & 15 \\
\hline 2MASS J04562935+3036115 & XEST 26-135 & $<\mathrm{M} 0$ & X-ray & 17 \\
\hline
\end{tabular}

Notes.

${ }^{a}$ Sources that are not in the 2MASS Point Source Catalog have been assigned coordinate-based identifications using IRAC images.

b Sources were selected for spectroscopy because they were candidate companions to known Taurus members ("comp", Kraus \& Hillenbrand 2007) or they were identified as candidate members with XMM-Newton ("X-ray", Scelsi et al. 2007) or Spitzer ("IR", this work).

${ }^{c}$ Mistakenly identified as FW Tau B by Hartmann et al. (2005a). White \& Ghez (2001) classified it as a probable background star based on the presence of $\mathrm{H} \alpha$ absorption. 


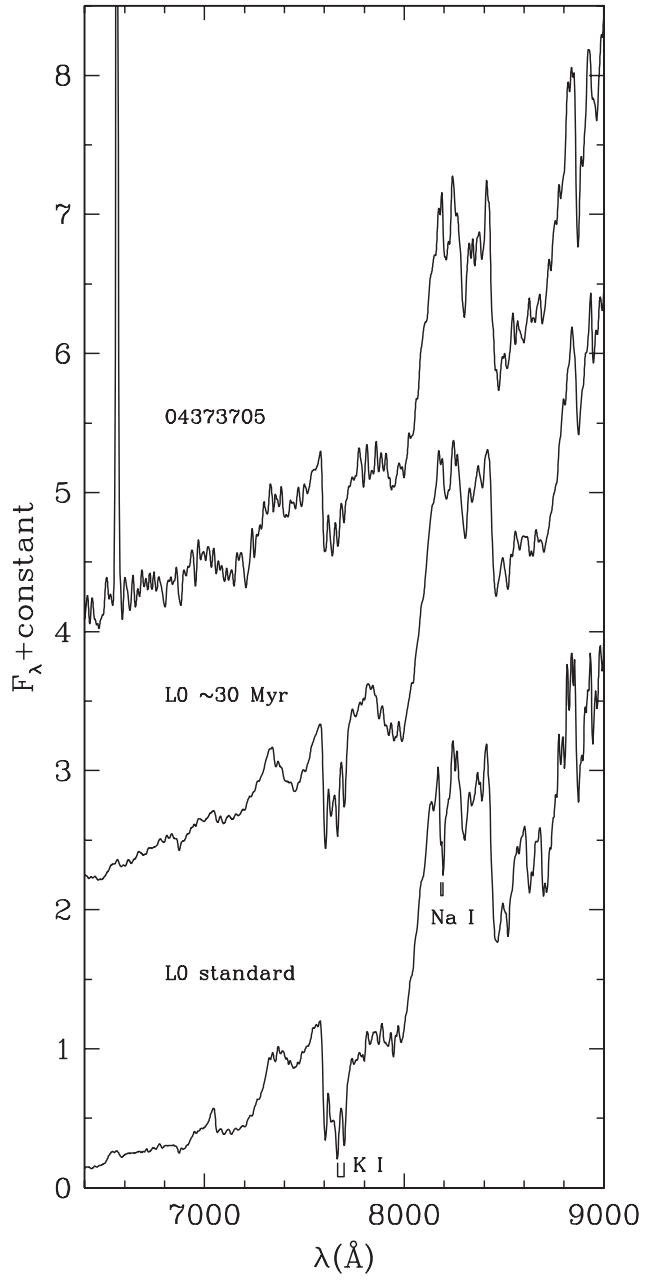

Figure 2. Optical spectrum of the new Taurus member 2MASS J04373705+2331080 compared to data for the young L0 dwarf 2MASS 01415823-4633574 (Kirkpatrick et al. 2006; Cruz et al. 2009) and the L0 standard 2MASS J03454316+2540233 (Kirkpatrick et al. 1999). The proper motion of 2MASS 01415823-4633574 from Faherty et al. (2009) is consistent with membership in the Tucana-Horologium association, which has an age of $\sim 30 \mathrm{Myr}$ (Torres et al. 2000; Zuckerman \& Song 2004). The data are displayed at a resolution of $13 \AA$ and are normalized at $7500 \AA$.

2009), and the bolometric corrections used by Luhman (2004a, 2007). The luminosities of 2MASS J04194657+2712552 (hereafter $2 \mathrm{M} 0419+2712$ ) and FU Tau B are based on $H$ and $K_{s}$, respectively, since reliable $J$-band measurements are unavailable. We have converted our spectral types to effective temperatures with the temperature scales from Schmidt-Kaler (1982) and Luhman et al. (2003b) for $<\mathrm{M} 0$ and $\geqslant \mathrm{M} 0$, respectively. As done by Luhman et al. (2008), we adopt a temperature of $2200 \mathrm{~K}$ for L0. The extinctions, effective temperatures, and bolometric luminosities are presented in Table 2. We cannot measure these parameters for 2MASS J04293209+2430597 because it lacks a spectral classification.

For comparison to the new Taurus members on the H-R diagram, we have compiled temperatures and luminosities for previously known members (see the Appendix). We exclude members that are anomalously faint for their spectral types because their luminosity estimates are probably unreliable. We treat multiple systems that are unresolved by 2MASS as single objects. We adopt extinctions that have been derived from our previous optical and near-IR spectroscopy (Luhman et al. 2003a; Luhman 2004c, 2006; Luhman et al. 2006) and additional unpublished $0.8-2.5 \mu \mathrm{m}$ spectra obtained with SpeX. For members that lack
Table 4

Measurements of Li Absorption

\begin{tabular}{lc}
\hline \hline \multicolumn{1}{c}{ Name } & $\begin{array}{c}W_{\lambda} \\
(\AA)\end{array}$ \\
\hline HQ Tau & $0.4 \pm 0.05$ \\
XEST 09-042 & $0.55 \pm 0.05$ \\
XEST 08-003 & $0.6 \pm 0.05$ \\
V409 Tau & $0.6 \pm 0.1$ \\
XEST 26-062 & $0.47 \pm 0.05$
\end{tabular}

spectroscopic data of this kind, we estimate extinctions from $J-H$ and $H-K_{s}$. Luminosities are based on measurements of $J$ from 2MASS when available. We adopt $J$ from multiplicity surveys for a few systems that are marginally resolved by 2MASS.

Our luminosity estimates for the new members XEST 11-078, 2MASS J04202583+2819237, 2MASS J04333905+2227207, and 2MASS J04202144+2813491 would place these stars well below the sequence of known Taurus members in an H-R diagram, which suggests that they may be seen in scattered light. Indeed, we demonstrate in the next section that 2MASS J04202144+2813491 has an edge-on disk. Because our calculated luminosities for these objects are probably not reliable, we do not report these estimates in Table 2 and do not plot these stars on the H-R diagram in Figure 7.

The temperatures and luminosities of the new and previously known members of Taurus (except for subluminous sources) are plotted on an H-R diagram in Figure 7 with the predictions of theoretical evolutionary models (Baraffe et al. 1998; Chabrier et al. 2000). LH $0429+17$ is included among the previously known members in Figure 7. A few of the new members have distinctive positions in the $\mathrm{H}-\mathrm{R}$ diagram. FU Tau A and $2 \mathrm{M}$ $0419+2712$ are the brightest known members of Taurus near their spectral types. The overluminous nature of the former was discussed by Luhman et al. (2009b). The high luminosity of $2 \mathrm{M} 0419+2712$ is consistent with the very early evolutionary stage that is implied by the shape of its spectral energy distribution (SED; Section 4.2.2). Meanwhile, the temperature and luminosity of the coolest known member of Taurus, $2 \mathrm{M}$ $0437+2331$, correspond to an age of $100 \mathrm{Myr}$ according to the evolutionary models, which is much older than expected for a member of Taurus. Stars with edge-on disks and nonmembers can appear to have very low luminosities on the H-R diagram. However, a disk does not appear to be present (Section 4.2.1) and the strengths of the gravity-sensitive lines of this object provide strong evidence of youth. Instead, the old isochronal age for this object is likely a reflection of errors in the adopted temperature scale and evolutionary models. This conclusion is based on the cluster sequence for Chamaeleon I, which falls along older model isochrones at spectral types later than M8 (Luhman et al. 2008). In fact, the position of the coolest known member of Chamaeleon I on the H-R diagram is very similar to that of $2 \mathrm{M} 0437+2331$. Thus, the luminosity of the latter is not anomalous when compared to other young late-type objects. If we estimate the mass of $2 \mathrm{M} 0437+2331$ based on its luminosity as done by Luhman et al. (2008) for the coolest member of Chamaeleon I, then we arrive at a value of 4-7 $M_{\text {Jup }}$ for an assumed age range of 1-3 Myr.

\subsection{Disks}

We can use the extensive mid-IR images of Taurus from Spitzer to determine whether the new Taurus members have circumstellar disks. Photometry from IRAC (3.6-8.0 $\mu \mathrm{m})$ and 


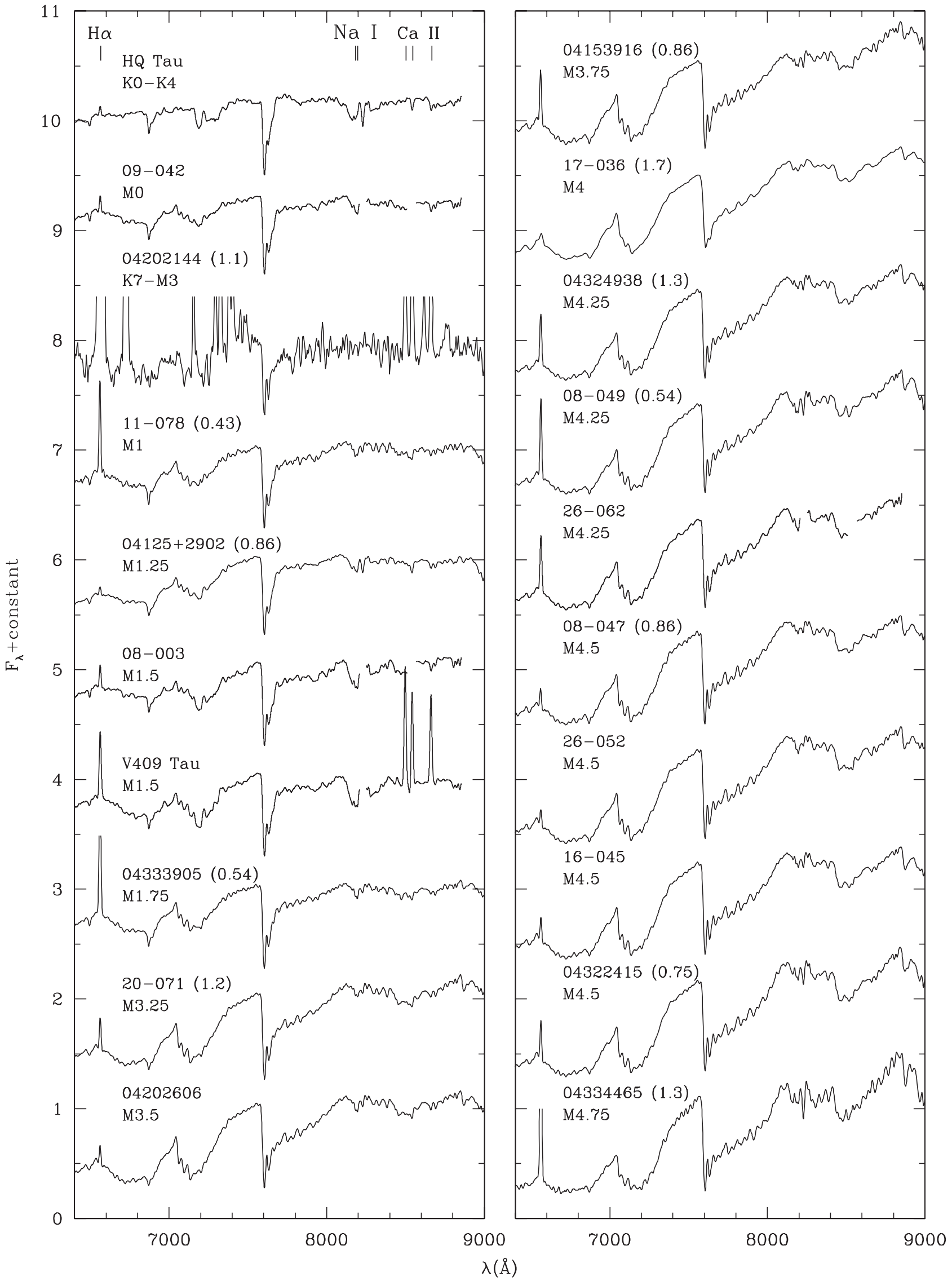

Figure 3. Optical spectra of new members of Taurus. The spectra have been corrected for extinction, which is quantified in parentheses by the magnitude difference of the reddening between 0.6 and $0.9 \mu \mathrm{m}(E(0.6-0.9))$. The data are displayed at a resolution of $13 \AA$ and are normalized at $7500 \AA$. 


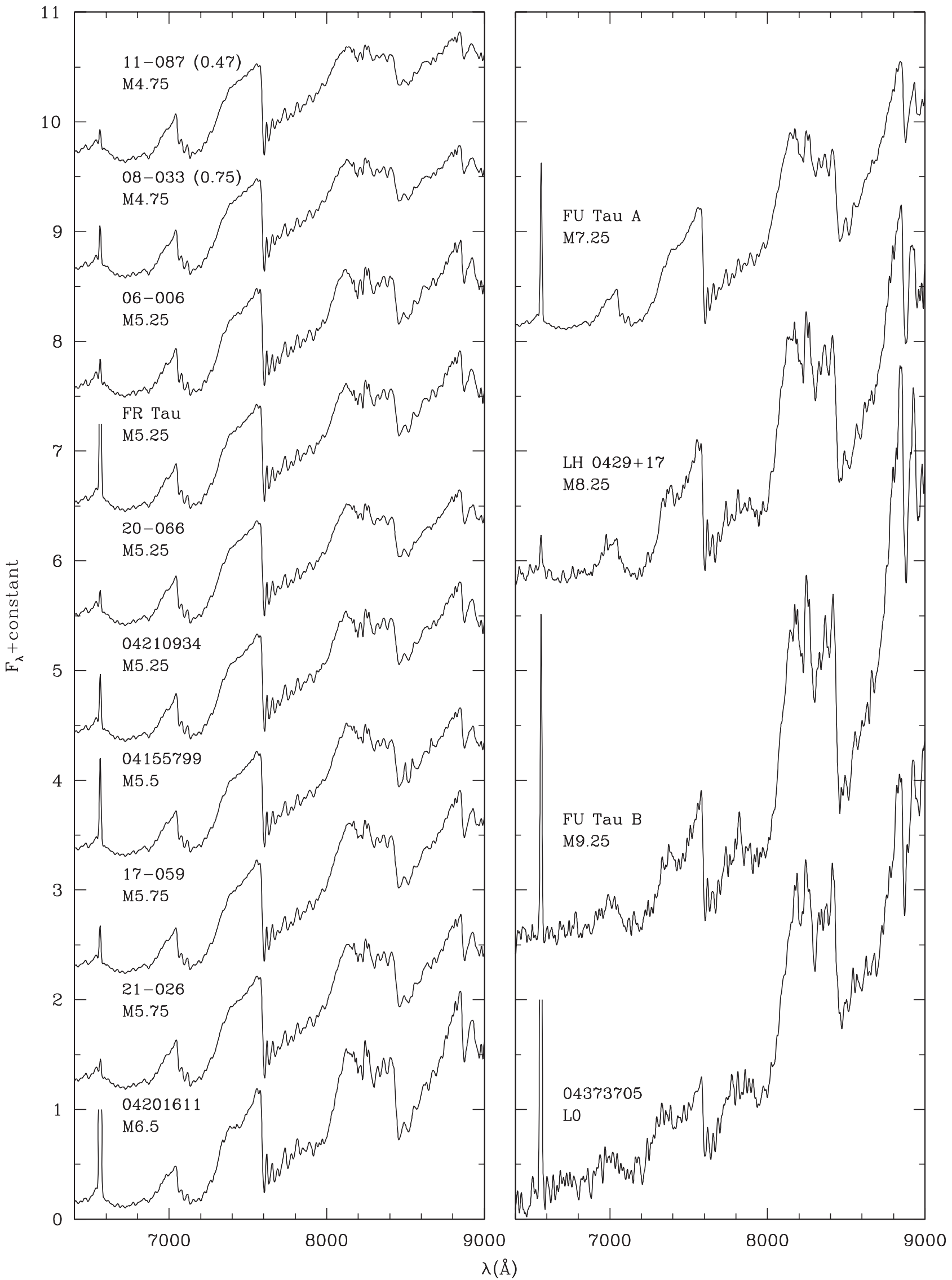

Figure 4. More optical spectra of new members of Taurus (see Figure 3) and the previously known member LH 0429+17 (Reid \& Hawley 1999). 


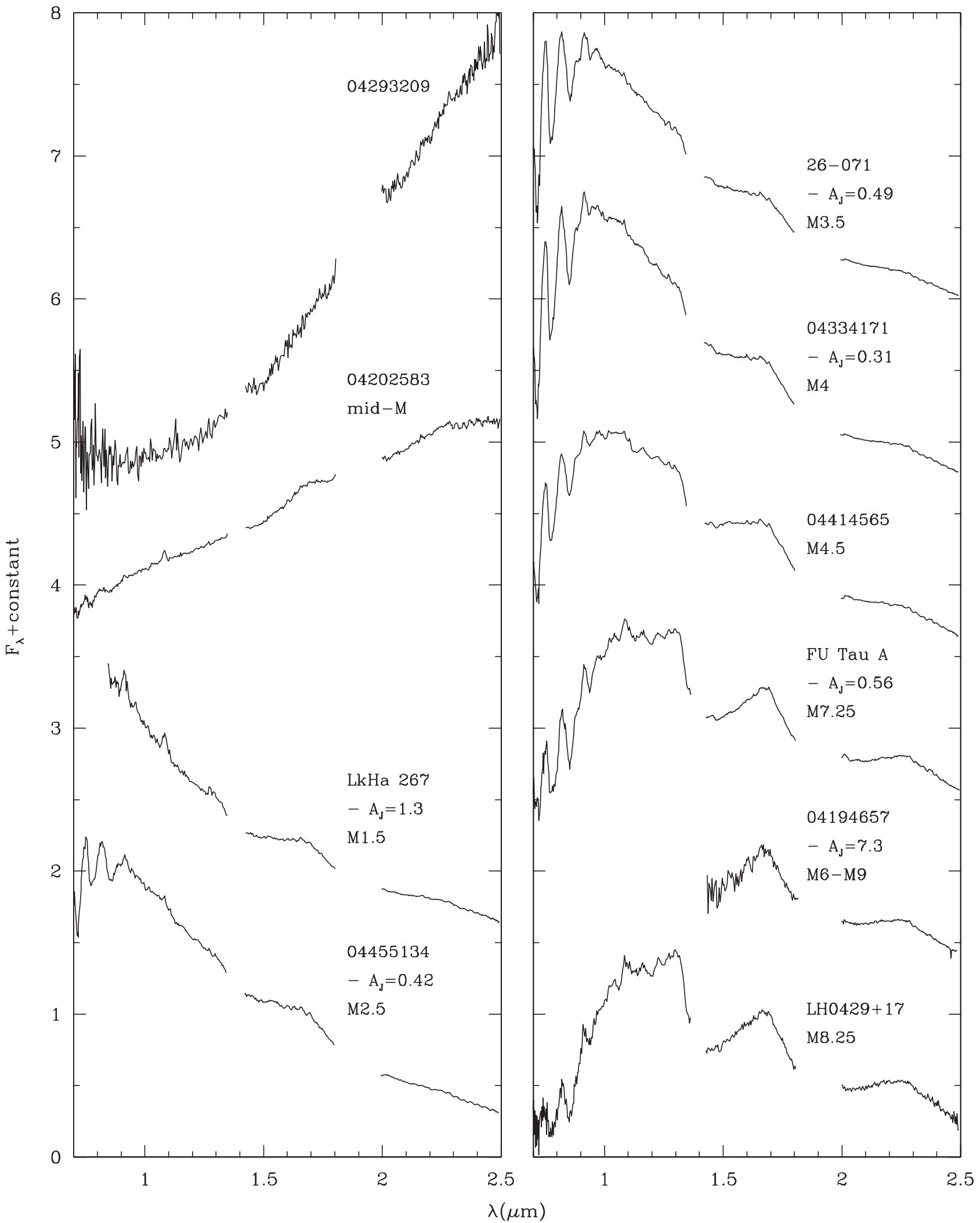

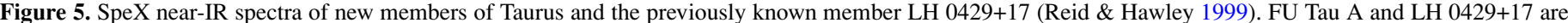

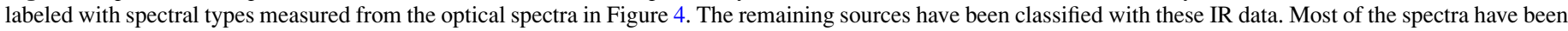

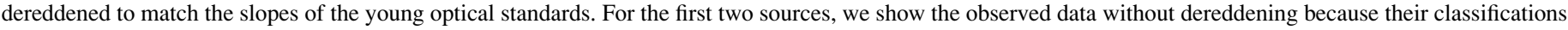
are uncertain. These data have a resolution of $R=100$ and are normalized at $1.68 \mu \mathrm{m}$.

MIPS $(24 \mu \mathrm{m})$ for the new members is presented by Luhman et al. (2009a), who measured photometry for all known members of Taurus that appear within Spitzer images of the region.
IRAC and MIPS data for the nonmembers in our spectroscopic sample are provided in Tables 5 and 6, respectively. Using the Spitzer photometry, Luhman et al. (2009a) have classified 


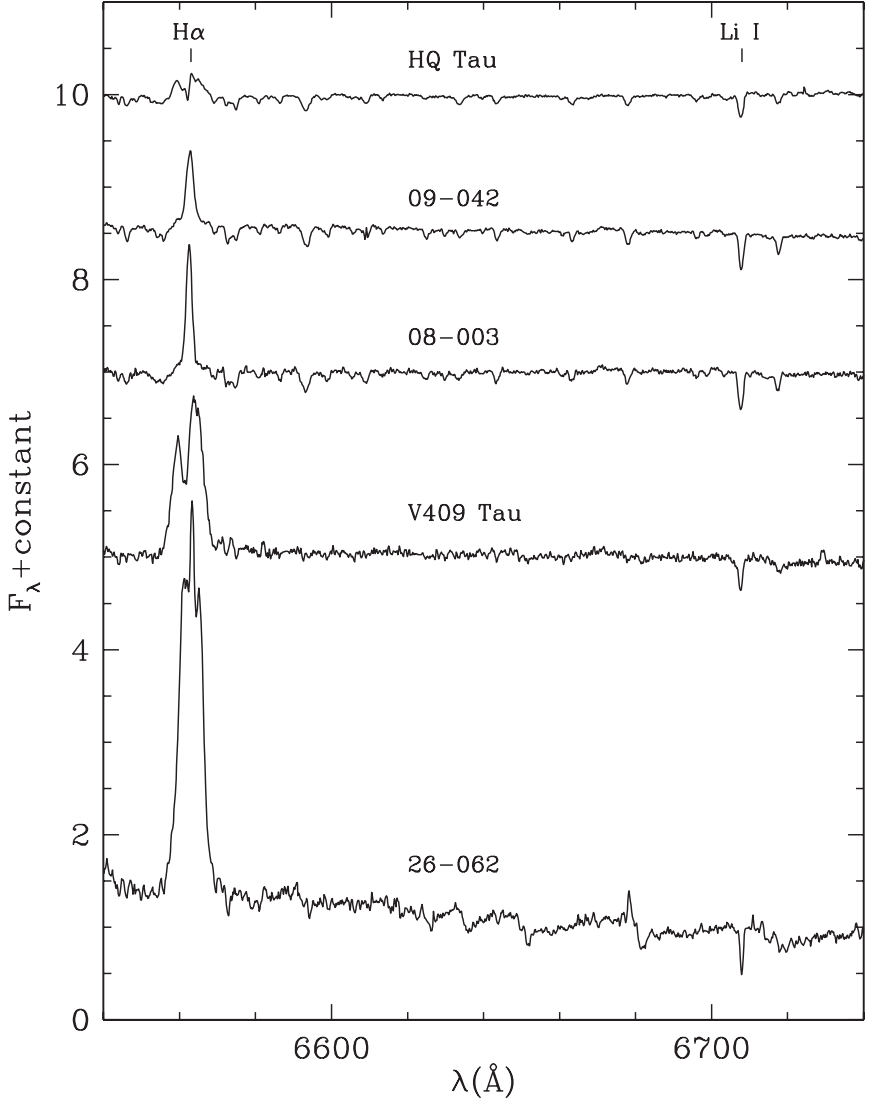

Figure 6. High-resolution spectra of $\mathrm{H} \alpha$ and $\mathrm{Li}$ I for new members of Taurus. The data have a resolution of $0.7 \AA$ and are normalized to the continuum near the Li I line.

each member of Taurus as class I, II, or III, which follows the standard classification scheme for the SEDs of young stars (Lada 1987). The 41 new members in our spectroscopic sample were identified as candidates through data from Spitzer (24), $X M M$-Newton (16), and a companion survey (1). Although the Spitzer members were selected based on red mid-IR colors, one of these sources $(2 \mathrm{M} 0437+2331)$ does not show evidence of a disk upon closer examination of its colors later in this section. All of the X-ray members have been observed by IRAC and MIPS, but these data are not yet publicly available for one object, XEST 06-006. XEST 11-078 and XEST 26-062 are class I and class II, respectively, while the remaining 13 $\mathrm{X}$-ray sources are class III. The candidate companion 2MASS $\mathrm{J} 04414565+2301580$ is class III as well. In the remainder of this section, we discuss in detail the evidence of disks for a few of the new members that are particularly notable.

\subsubsection{L-type Brown Dwarf: No Disk}

$2 \mathrm{M} 0437+2331$ is the coolest known member of Taurus and may be one of the least massive known brown dwarfs. It was selected for spectroscopy based on red IRAC colors that suggested the presence of a disk (it was not detected by MIPS). To reliably determine whether $2 \mathrm{M} 0437+2331$ does indeed have a disk, we must compare its colors to those of stellar photospheres. For these comparison sources, we select all known late-type members of Taurus and young late-M and $\mathrm{L}$ dwarfs in the solar neighborhood (Kirkpatrick et al. 2006; Cruz et al. 2009) that have been observed by IRAC. The IRAC data for the Taurus members are taken from Luhman et al. (2009a). We have measured photometry for young field dwarfs observed in Spitzer programs 284 (K. Cruz) and 30540 (J. Houck) with the same methods that were employed for the Taurus data. These data are presented in Table 7. We show [3.6]-[5.8] and [3.6]-[8.0] versus spectral type for $2 \mathrm{M} 0437+2331$, other Taurus members, and the young field dwarfs in Figure 8. These data form two distinct groups, a narrow sequence of stellar photospheres and a broader distribution of redder sources that have disks. $2 \mathrm{M} 0437+2331$ is only slightly redder than the photospheric sequences ( $\sim 0.2 \mathrm{mag})$ and does not exhibit color excesses as large as those found among the Taurus members that have disks $(\sim 0.5-1.5 \mathrm{mag})$. Thus, we do not find significant evidence of a disk for this object. As demonstrated in Figure 8, the photospheric IRAC colors become redder from $\mathrm{M}$ to $\mathrm{L}$ types among young objects, which explains why 2M $0437+2331$ was identified as a possible disk-bearing object through our IRAC color criteria. Because young stars and brown dwarfs that lack IRAC excesses rarely exhibit evidence of accretion in $\mathrm{H} \alpha$ (Mohanty et al. 2005; Muzerolle et al. 2003, 2005; Hartmann et al. 2005a; Guieu et al. 2007; Luhman et al. 2006, 2008), the $\mathrm{H} \alpha$ emission in the spectrum of $2 \mathrm{M} 0437+2331$ probably arises from the stellar chromosphere rather than accretion.

\subsubsection{Candidate Class I Brown Dwarf}

The IR spectrum of $2 \mathrm{M} 0419+2712$ has strong $\mathrm{H}_{2} \mathrm{O}$ absorption and is highly reddened $\left(A_{J}=7.6\right)$, making it the most heavily obscured late-type member of Taurus found to date. To further investigate the nature of this object, we have constructed its SED in Figure 9 with photometry from 2MASS, IRAC, and MIPS and our near-IR spectrum, which was fluxcalibrated with the 2MASS data. The Taurus member KPNO 5 is similar to $2 \mathrm{M} 0419+2712$ in spectral type and does not have mid-IR excess emission (Briceño et al. 2002; Hartmann et al. 2005a; Luhman et al. 2006). Therefore, we include the SED of KPNO 5 in Figure 9 as an estimate of the stellar photosphere of 2M 0419+2712. The SED of KPNO 5 consists of data from 2MASS, SpeX (Muench et al. 2007), and Spitzer (Luhman et al. 2009a), and has been reddened by $A_{J}=7.3$ to match the reddening of $2 \mathrm{M} 0419+2712$ (KPNO 5 has $A_{J} \sim 0.3$ ). We use the reddening laws from Rieke \& Lebofsky (1985) and Flaherty et al. (2007). As shown in Figure 9, 2M 0419+2712 exhibits significant excess emission relative to KPNO 5 beyond $3 \mu \mathrm{m}$. The SED of $2 \mathrm{M} 0419+2712$ is roughly flat from $2-24 \mu \mathrm{m}$, which is suggestive of an evolved class I source (star+disk+envelope) or a very young class II object (star+disk). Thus, 2M 0419+2712 may offer a rare opportunity for studying a brown dwarf in the protostellar phase. A definitive classification of the evolutionary stage of this object will require additional observations, such as mid-IR spectroscopy (Furlan et al. 2008).

\subsubsection{Candidate Transitional Disk}

Most of the candidate members that we identified with Spitzer photometry have red colors in all available bands beyond $3 \mu \mathrm{m}$. However, one of the confirmed members from that sample, IRAS $04125+2902$, is red only at $24 \mu \mathrm{m}$. To illustrate the distinctive colors of this star, we compare its SED to the average SED of early-M diskless stars in Chamaeleon I and Taurus in Figure 9 (Luhman et al. 2008, 2009a). The data for IRAS 04125+2902 agree well with the photospheric SED in the 2MASS and IRAC bands, but they show significant excess emission at $24 \mu \mathrm{m}$. An SED of this kind indicates the presence of a disk with an inner hole, which is known as a transitional disk (Calvet et al. 2002, 2005; D'Alessio et al. 2005). Mid-IR spectroscopy and millimeter imaging of this disk are needed for a detailed 


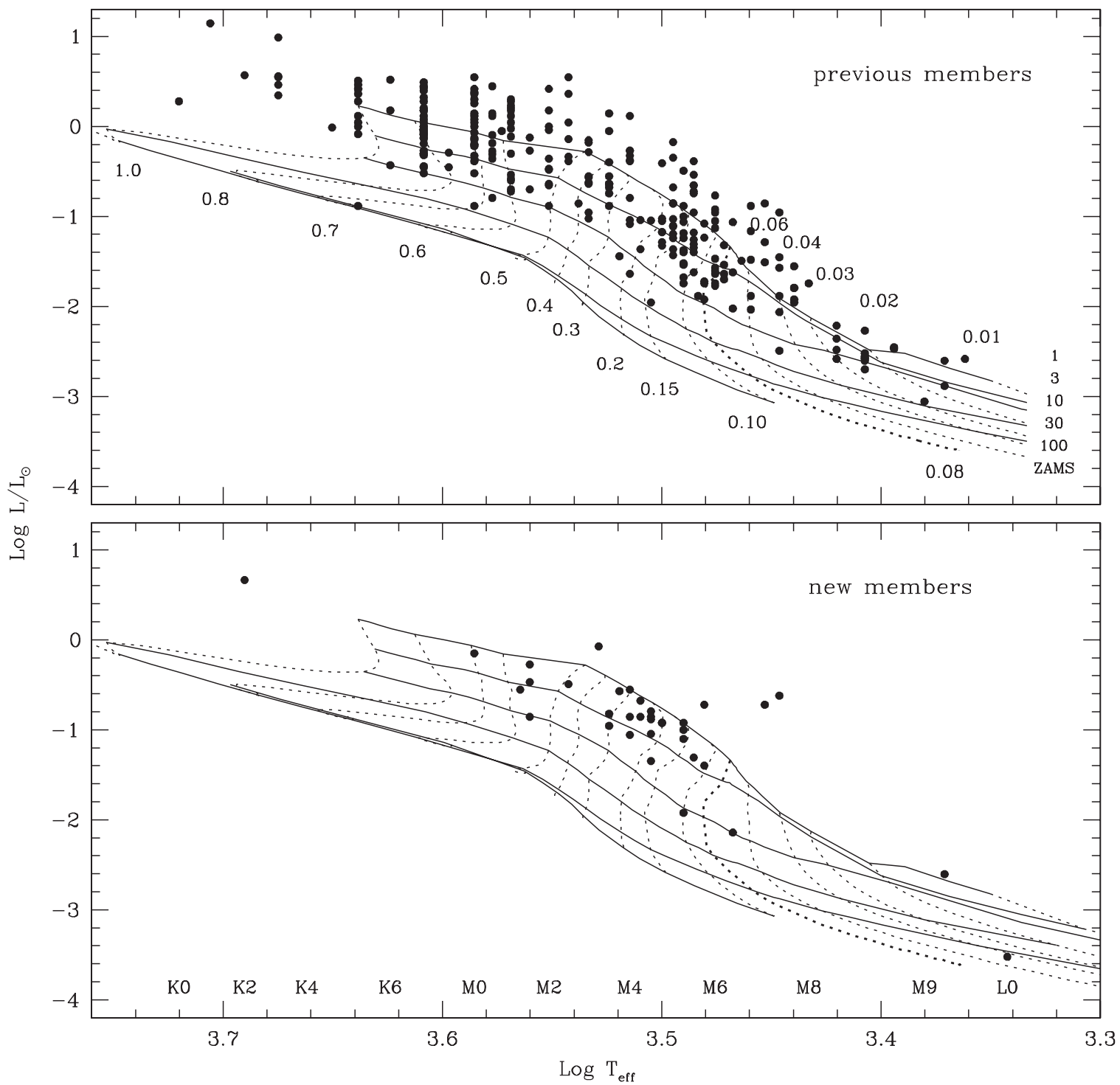

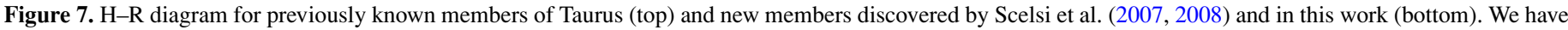

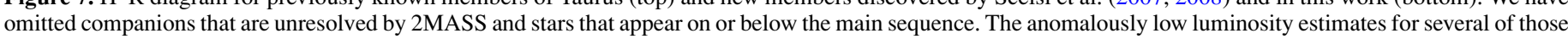

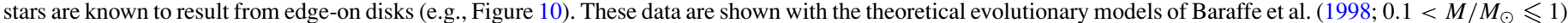
and Chabrier et al. $\left(2000 ; M / M_{\odot} \leqslant 0.1\right)$, where the mass tracks (dotted lines) and isochrones (solid lines) are labeled in units of $M_{\odot}$ and Myr, respectively.

characterization of the inner hole (Espaillat et al. 2007a, 2007b; Furlan et al. 2007; Brown et al. 2008; Dutrey et al. 2008; Hughes et al. 2007, 2009).

\subsubsection{Edge-on Disk}

As noted in the previous section, a few of the new Taurus members are much fainter at optical and near-IR wavelengths than most other members with similar spectral types, indicating that they may have edge-on disks. We have investigated this possibility by inspecting images of these stars that are available in the data archives of various observatories and wide-field surveys. In optical images from MegaPrime/ MegaCam at the Canada-France-Hawaii Telescope (CFHT), 2MASS J04202144+2813491 does indeed exhibit a clear signature of an edge-on disk in the form of two lobes of extended emission that are separated by a dark lane, as shown in Figure 10 . The radius of the disk is $2 . .5$, corresponding to $350 \mathrm{AU}$ at the distance of Taurus. After comparing CFHT images that were collected on different nights, we find that the scattered light from the disk experienced significant variability over a period of a few days (see Figure 10). Similar variability has been detected in the scattered light from HH 30 (Burrows et al. 1996; Watson \& Stapelfeldt 2007). The discovery of 2MASS J04202144+2813491 illustrates the utility of Spitzer imaging for finding edge-on disks, which can be overlooked through other types of surveys for young stars (Luhman \& Muench 2008).

\subsection{Companions}

A few of the new Taurus members have candidate companions at wide separations. 2MASS J04414565+2301580 is located 12" from the known member 2MASS J04414489+2301513 (M8.5 Luhman 2006) and was identified as a possible companion by Kraus \& Hillenbrand (2007). We have confirmed its youth and membership in Taurus through spectroscopy. Our spectral classification of M4-M5 for 2MASS J04414565+2301580 is consistent with that expected for a coeval companion to 2MASS J04414489+2301513 based on the evolutionary models of Baraffe et al. (1998). While performing spectroscopy on FU 
Table 5

IRAC Photometry for Nonmembers

\begin{tabular}{|c|c|c|c|c|c|}
\hline ID & {$[3.6]$} & {$[4.5]$} & {$[5.8]$} & {$[8.0]$} & Date \\
\hline 2MASS J04124858+2749563 & $11.40 \pm 0.02$ & $10.87 \pm 0.02$ & $10.32 \pm 0.03$ & $9.14 \pm 0.03$ & 2007 Mar 29 \\
\hline \multirow{2}{*}{ 2MASS J04141588+2818181 } & $12.36 \pm 0.02$ & $12.11 \pm 0.02$ & $12.13 \pm 0.04$ & $12.10 \pm 0.04$ & 2005 Feb 19 \\
\hline & $12.21 \pm 0.02$ & $12.07 \pm 0.02$ & $12.10 \pm 0.04$ & $12.00 \pm 0.04$ & 2007 Mar 29 \\
\hline \multirow[t]{2}{*}{ 2MASS J04144294+2821105 } & $11.75 \pm 0.02$ & $11.62 \pm 0.02$ & $11.54 \pm 0.03$ & $11.56 \pm 0.04$ & 2005 Feb 19 \\
\hline & $11.70 \pm 0.02$ & $11.77 \pm 0.02$ & $11.49 \pm 0.03$ & $11.46 \pm 0.04$ & $2007 \operatorname{Mar} 29$ \\
\hline 2MASS J04164774+2408242 & $13.84 \pm 0.03$ & $13.36 \pm 0.03$ & $13.12 \pm 0.05$ & $12.26 \pm 0.04$ & 2007 Mar 30 \\
\hline 2MASS J04170711+2408041 & $13.93 \pm 0.03$ & $13.72 \pm 0.03$ & $13.53 \pm 0.06$ & $12.63 \pm 0.06$ & 2007 Mar 30 \\
\hline 2MASS J04180338+2440096 & $10.04 \pm 0.02$ & $10.03 \pm 0.02$ & $10.03 \pm 0.03$ & $9.83 \pm 0.03$ & 2007 Mar 30 \\
\hline 2MASS J04180674+2904015 & $13.74 \pm 0.03$ & $13.48 \pm 0.03$ & $13.51 \pm 0.06$ & $12.61 \pm 0.06$ & 2007 Mar 29 \\
\hline 2MASS J04182321+2519280 & $11.76 \pm 0.02$ & $11.06 \pm 0.02$ & $10.45 \pm 0.03$ & $9.62 \pm 0.03$ & 2007 Mar 30 \\
\hline \multirow[t]{2}{*}{ 2MASS J04190125+2837101 } & out & $10.69 \pm 0.02$ & out & $10.65 \pm 0.03$ & 2005 Feb 19 \\
\hline & $10.68 \pm 0.02$ & $10.67 \pm 0.02$ & $10.68 \pm 0.03$ & $10.65 \pm 0.03$ & 2007 Mar 29 \\
\hline \multirow[t]{2}{*}{ 2MASS J04190689+2826090 } & $12.52 \pm 0.02$ & $12.42 \pm 0.02$ & $12.38 \pm 0.04$ & $12.31 \pm 0.04$ & 2005 Feb 19 \\
\hline & $12.55 \pm 0.02$ & $12.43 \pm 0.02$ & $12.39 \pm 0.04$ & $12.25 \pm 0.04$ & 2005 Feb 21 \\
\hline 2MASS J04191612+2750481 & $11.84 \pm 0.02$ & $11.15 \pm 0.02$ & $10.48 \pm 0.03$ & $9.39 \pm 0.03$ & 2005 Feb 21 \\
\hline 2MASS J04214372+2647225 & $8.81 \pm 0.02$ & $8.85 \pm 0.02$ & $8.77 \pm 0.03$ & $8.77 \pm 0.03$ & 2007 Oct 17 \\
\hline \multirow[t]{2}{*}{ 2MASS J04221295+2546598 } & $12.60 \pm 0.02$ & $11.81 \pm 0.02$ & $11.16 \pm 0.03$ & $10.37 \pm 0.03$ & 2006 Sep 28 \\
\hline & $12.54 \pm 0.02$ & $11.80 \pm 0.02$ & $11.17 \pm 0.03$ & $10.32 \pm 0.03$ & 2007 Mar 30 \\
\hline 2MASS J04221918+2348005 & $14.08 \pm 0.03$ & $13.59 \pm 0.03$ & $13.42 \pm 0.06$ & $12.25 \pm 0.05$ & 2007 Mar 30 \\
\hline 2MASS J04222559+2812332 & $8.54 \pm 0.02$ & $8.54 \pm 0.02$ & $8.55 \pm 0.03$ & $8.50 \pm 0.03$ & 2005 Feb 21 \\
\hline 2MASS J04222718+2659512 & $13.92 \pm 0.02$ & $13.75 \pm 0.03$ & $13.77 \pm 0.07$ & $\ldots$ & 2007 Oct 17 \\
\hline 2MASS J04223441+2457186 & $14.77 \pm 0.03$ & $14.13 \pm 0.03$ & $13.64 \pm 0.06$ & $12.87 \pm 0.06$ & 2007 Mar 30 \\
\hline 2MASS J04224865+2823005 & $13.17 \pm 0.02$ & $13.07 \pm 0.02$ & $13.03 \pm 0.05$ & $13.23 \pm 0.07$ & 2005 Feb 21 \\
\hline 2MASS J04263497+2608161 & $13.09 \pm 0.02$ & $12.55 \pm 0.02$ & $11.66 \pm 0.03$ & $9.63 \pm 0.03$ & 2005 Feb 22 \\
\hline \multirow[t]{2}{*}{ 2MASS J04275871+2611062 } & $9.77 \pm 0.02$ & out & $9.71 \pm 0.03$ & out & 2004 Mar 7 \\
\hline & $9.80 \pm 0.02$ & $9.85 \pm 0.02$ & $9.84 \pm 0.03$ & $9.75 \pm 0.03$ & 2005 Feb 22 \\
\hline 2MASS J04285844+2436492 & $10.63 \pm 0.02$ & $10.54 \pm 0.02$ & $10.47 \pm 0.03$ & $10.47 \pm 0.03$ & 2005 Feb 20 \\
\hline 2MASS J04292083+2742074 & $6.66 \pm 0.02$ & $6.52 \pm 0.02$ & $6.18 \pm 0.03$ & $4.98 \pm 0.03$ & 2005 Feb 24 \\
\hline \multirow[t]{3}{*}{ 2MASS J04292887+2616483 } & $9.22 \pm 0.02$ & $9.21 \pm 0.02$ & $9.12 \pm 0.03$ & $9.08 \pm 0.03$ & $2004 \operatorname{Mar} 7$ \\
\hline & $9.21 \pm 0.02$ & $9.24 \pm 0.02$ & $9.10 \pm 0.03$ & $9.12 \pm 0.03$ & 2005 Feb 24 \\
\hline & $9.22 \pm 0.02$ & $9.15 \pm 0.02$ & $9.15 \pm 0.03$ & $9.07 \pm 0.03$ & 2006 Mar 25 \\
\hline \multirow[t]{2}{*}{ 2MASS J04293623+2634238 } & out & $10.30 \pm 0.02$ & out & $10.23 \pm 0.03$ & 2004 Mar 7 \\
\hline & $10.38 \pm 0.02$ & $10.33 \pm 0.02$ & $10.26 \pm 0.03$ & $10.27 \pm 0.03$ & 2005 Feb 24 \\
\hline 2MASS J04301702+2622264 & $10.63 \pm 0.02$ & $10.57 \pm 0.02$ & $10.58 \pm 0.03$ & $10.52 \pm 0.03$ & 2005 Feb 24 \\
\hline 2MASS J04302526+2602566 & $10.04 \pm 0.02$ & $10.01 \pm 0.02$ & $10.04 \pm 0.03$ & $9.97 \pm 0.03$ & 2005 Feb 24 \\
\hline 2MASS J04302710+2807073 & $10.82 \pm 0.02$ & $9.68 \pm 0.02$ & $8.70 \pm 0.03$ & $7.61 \pm 0.03$ & $2005 \mathrm{Feb} 24$ \\
\hline 2MASS J04304153+2430416 & $9.20 \pm 0.02$ & $9.21 \pm 0.02$ & $9.17 \pm 0.03$ & $9.19 \pm 0.03$ & 2005 Feb 24 \\
\hline \multirow[t]{2}{*}{ 2MASS J04314419+2813170 } & $14.04 \pm 0.03$ & $13.92 \pm 0.03$ & $13.97 \pm 0.07$ & $12.83 \pm 0.06$ & 2005 Feb 20 \\
\hline & $13.96 \pm 0.03$ & $13.87 \pm 0.03$ & $13.71 \pm 0.08$ & $12.88 \pm 0.06$ & 2005 Feb 24 \\
\hline 2MASS J04314634+2558404 & $12.12 \pm 0.02$ & $12.12 \pm 0.02$ & $12.07 \pm 0.04$ & $12.06 \pm 0.03$ & 2005 Feb 24 \\
\hline 2MASS J04315860+1818408 & $9.10 \pm 0.02$ & $9.09 \pm 0.02$ & $9.14 \pm 0.03$ & $9.03 \pm 0.03$ & 2004 Oct 7 \\
\hline \multirow[t]{2}{*}{ 2MASS J04322689+1818230 } & $11.46 \pm 0.02$ & $11.45 \pm 0.02$ & $11.51 \pm 0.03$ & $11.36 \pm 0.03$ & 2004 Oct 7 \\
\hline & $11.47 \pm 0.02$ & out & $11.38 \pm 0.03$ & out & 2005 Feb 19 \\
\hline 2MASS J04322946+1814002 & $12.68 \pm 0.02$ & $12.54 \pm 0.02$ & $12.17 \pm 0.04$ & $10.90 \pm 0.03$ & 2004 Oct 7 \\
\hline \multirow[t]{3}{*}{ IRAC J04323521+2420213 } & $13.58 \pm 0.03$ & $12.56 \pm 0.02$ & $11.73 \pm 0.03$ & $10.76 \pm 0.03$ & 2004 Oct 7 \\
\hline & $13.54 \pm 0.02$ & $12.54 \pm 0.02$ & $11.69 \pm 0.04$ & $10.77 \pm 0.03$ & 2005 Feb 20 \\
\hline & $13.55 \pm 0.03$ & $12.65 \pm 0.03$ & $11.72 \pm 0.04$ & $10.80 \pm 0.03$ & 2005 Feb 24 \\
\hline \multirow[t]{2}{*}{ 2MASS J04323605+2552225 } & $13.84 \pm 0.02$ & $13.80 \pm 0.03$ & $13.74 \pm 0.07$ & $13.52 \pm 0.09$ & 2004 Mar 7 \\
\hline & $13.89 \pm 0.02$ & $13.80 \pm 0.02$ & $13.84 \pm 0.06$ & $13.48 \pm 0.07$ & 2005 Feb 24 \\
\hline \multirow[t]{3}{*}{ 2MASS J04323949+2427043 } & $11.94 \pm 0.02$ & $11.86 \pm 0.02$ & $11.76 \pm 0.03$ & $11.77 \pm 0.03$ & 2004 Oct 7 \\
\hline & $11.97 \pm 0.02$ & $11.82 \pm 0.02$ & $11.68 \pm 0.03$ & $11.71 \pm 0.04$ & 2005 Feb 24 \\
\hline & $11.93 \pm 0.02$ & $11.80 \pm 0.02$ & $11.77 \pm 0.03$ & $11.69 \pm 0.03$ & 2006 Mar 26 \\
\hline 2MASS J04325921+2430403 & $14.00 \pm 0.02$ & out & $13.54 \pm 0.05$ & out & 2004 Oct 7 \\
\hline & $13.98 \pm 0.03$ & $13.74 \pm 0.03$ & $13.44 \pm 0.06$ & $11.36 \pm 0.04$ & 2005 Feb 24 \\
\hline 2MASS J04332491+2559262 & $13.08 \pm 0.02$ & $13.01 \pm 0.02$ & $13.10 \pm 0.05$ & $12.98 \pm 0.05$ & 2005 Feb 24 \\
\hline 2MASS J04333301+2252521 & $14.07 \pm 0.03$ & out & $13.81 \pm 0.07$ & out & 2005 Feb 24 \\
\hline & $13.98 \pm 0.02$ & $14.02 \pm 0.03$ & $14.17 \pm 0.10$ & $\cdots$ & 2007 Apr 3 \\
\hline 2MASS J04333746+2609550 & $12.82 \pm 0.04$ & $12.63 \pm 0.08$ & $12.58 \pm 0.05$ & $\cdots$ & $2004 \operatorname{Mar} 7$ \\
\hline & $12.78 \pm 0.03$ & $12.71 \pm 0.05$ & $12.61 \pm 0.05$ & $\ldots$ & 2005 Feb 24 \\
\hline 2MASS J04335562+2425016 & $8.78 \pm 0.02$ & $8.83 \pm 0.02$ & $8.85 \pm 0.03$ & $8.76 \pm 0.03$ & 2005 Feb 24 \\
\hline 2MASS J04341498+2826124 & $13.83 \pm 0.02$ & $13.75 \pm 0.03$ & $13.73 \pm 0.06$ & $13.11 \pm 0.06$ & 2005 Feb 20 \\
\hline 2MASS J04343322+2602403 & $10.06 \pm 0.02$ & $10.03 \pm 0.02$ & $9.97 \pm 0.03$ & $10.01 \pm 0.03$ & 2005 Feb 22 \\
\hline 2MASS J04345164+2404426 & $11.63 \pm 0.02$ & $11.54 \pm 0.02$ & $11.50 \pm 0.03$ & $11.49 \pm 0.03$ & 2006 Mar 25 \\
\hline & $11.64 \pm 0.02$ & $11.55 \pm 0.02$ & $11.58 \pm 0.03$ & $11.52 \pm 0.03$ & 2007 Oct 16 \\
\hline 2MASS J04345973+2807017 & $14.34 \pm 0.03$ & $13.95 \pm 0.03$ & $13.71 \pm 0.06$ & $13.15 \pm 0.1$ & 2005 Feb 20 \\
\hline & $14.21 \pm 0.03$ & $13.91 \pm 0.03$ & $13.79 \pm 0.07$ & $13.04 \pm 0.1$ & 2007 Oct 16 \\
\hline
\end{tabular}


Table 5

(Continued)

\begin{tabular}{lccccc}
\hline \hline \multicolumn{1}{c}{ ID } & {$[3.6]$} & {$[4.5]$} & {$[5.8]$} & {$[8.0]$} & Date \\
\hline 2MASS J04351316+2259205 & out & $9.91 \pm 0.02$ & out & $9.84 \pm 0.03$ & $2005 \mathrm{Feb} 20$ \\
& $9.91 \pm 0.02$ & out & $9.84 \pm 0.03$ & out & $2005 \mathrm{Feb} 24$ \\
& $9.95 \pm 0.02$ & $9.90 \pm 0.02$ & $9.86 \pm 0.03$ & $9.84 \pm 0.03$ & $2007 \mathrm{Apr} \mathrm{3}$ \\
2MASS J04353651+2304590 & $14.52 \pm 0.03$ & $14.39 \pm 0.03$ & $14.55 \pm 0.12$ & $\ldots$ & $2005 \mathrm{Feb} 21$ \\
& out & $14.40 \pm 0.04$ & out & $\ldots$ & $2007 \mathrm{Apr} 3$ \\
2MASS J04354076+2411211 & $12.59 \pm 0.03$ & $12.34 \pm 0.04$ & $\ldots$ & $\ldots$ & $2005 \mathrm{Feb} 20$ \\
& $12.60 \pm 0.03$ & $12.29 \pm 0.05$ & $\ldots$ & $\ldots$ & $2005 \mathrm{Feb} 21$ \\
& $12.52 \pm 0.02$ & $12.26 \pm 0.04$ & $\ldots$ & $\ldots$ & $2006 \mathrm{Mar} 25$ \\
2MASS J04400363+2553547 & $7.55 \pm 0.02$ & out & $7.33 \pm 0.03$ & out & $2004 \mathrm{Mar} 7$ \\
& $7.54 \pm 0.02$ & $7.42 \pm 0.02$ & $7.39 \pm 0.03$ & $7.34 \pm 0.03$ & $2005 \mathrm{Feb} 22$ \\
2MASS J04403912+2540024 & $13.64 \pm 0.02$ & $13.51 \pm 0.03$ & $13.49 \pm 0.06$ & $13.54 \pm 0.09$ & $2007 \mathrm{Oct} 16$ \\
IRAC J04412575+2543492 & $13.73 \pm 0.02$ & $12.39 \pm 0.02$ & $11.22 \pm 0.03$ & $10.22 \pm 0.03$ & $2004 \mathrm{Oct} 08$ \\
& $13.76 \pm 0.02$ & $12.38 \pm 0.02$ & $11.21 \pm 0.03$ & $10.25 \pm 0.03$ & $2005 \mathrm{Feb} 23$ \\
2MASS J04415577+2302532 & $12.86 \pm 0.02$ & $12.24 \pm 0.02$ & $11.64 \pm 0.03$ & $10.40 \pm 0.03$ & $2007 \mathrm{Mar} 28$ \\
2MASS J04420376+2519533 & $13.22 \pm 0.02$ & $13.16 \pm 0.02$ & $13.17 \pm 0.05$ & $12.99 \pm 0.06$ & $2005 \mathrm{Feb} 23$ \\
2MASS J04455482+2408435 & $12.16 \pm 0.02$ & $11.46 \pm 0.02$ & $10.44 \pm 0.03$ & $8.67 \pm 0.03$ & $2007 \mathrm{Oct} 16$ \\
2MASS J04455704+2440423 & $13.03 \pm 0.02$ & $12.91 \pm 0.02$ & $12.63 \pm 0.04$ & $12.08 \pm 0.04$ & $2005 \mathrm{Feb} 24$ \\
2MASS J04553844+3031465 & $10.03 \pm 0.02$ & $10.09 \pm 0.02$ & $10.00 \pm 0.03$ & $10.04 \pm 0.03$ & $2004 \mathrm{Feb} 14$ \\
& $10.06 \pm 0.02$ & out & $10.02 \pm 0.03$ & out & $2005 \mathrm{Feb} 20$
\end{tabular}

Note. Entries of "..." and "out" indicate measurements that are absent because of nondetection and a position outside the field of view of the camera, respectively.

Table 6

MIPS $24 \mu \mathrm{m}$ Photometry for Nonmembers

\begin{tabular}{|c|c|c|}
\hline ID & {$[24]$} & Date \\
\hline 2MASS J04124858+2749563 & $6.46 \pm 0.04$ & 2007 Feb 27 \\
\hline 2MASS J04164774+2408242 & $8.98 \pm 0.11$ & 2007 Feb 26 \\
\hline 2MASS J04180338+2440096 & $7.20 \pm 0.05$ & 2007 Feb 23 \\
\hline 2MASS J04182321+2519280 & $6.17 \pm 0.04$ & 2007 Feb 23 \\
\hline \multirow[t]{2}{*}{ 2MASS J04191612+2750481 } & $5.25 \pm 0.04$ & 2005 Feb 28 \\
\hline & $5.28 \pm 0.04$ & 2007 Oct 28 \\
\hline \multirow{2}{*}{ 2MASS J04214372+2647225 } & $8.47 \pm 0.11$ & 2007 Feb 28 \\
\hline & $8.99 \pm 0.10$ & 2007 Oct 28 \\
\hline \multirow[t]{2}{*}{ 2MASS J04221295+2546598 } & $7.25 \pm 0.04$ & 2004 Sep 25 \\
\hline & $7.20 \pm 0.05$ & 2007 Feb 28 \\
\hline 2MASS J04221918+2348005 & $8.67 \pm 0.18$ & 2007 Feb 28 \\
\hline 2MASS J04222559+2812332 & $8.39 \pm 0.09$ & 2005 Feb 27 \\
\hline \multirow{2}{*}{ 2MASS J04263497+2608161 } & $5.34 \pm 0.04$ & 2005 Feb 28 \\
\hline & $5.38 \pm 0.04$ & 2005 Mar 1 \\
\hline 2MASS J04292083+2742074 & $3.05 \pm 0.04$ & 2005 Mar 2 \\
\hline 2MASS J04302710+2807073 & $3.68 \pm 0.04$ & 2005 Mar 1 \\
\hline \multirow{2}{*}{ 2MASS J04304153+2430416 } & $8.90 \pm 0.10$ & 2005 Feb 26 \\
\hline & $8.85 \pm 0.12$ & 2005 Mar 2 \\
\hline \multirow[t]{3}{*}{ 2MASS J04315860+1818408 } & $8.89 \pm 0.14$ & 2004 Feb 20 \\
\hline & $9.10 \pm 0.08$ & 2004 Sep 25 \\
\hline & $9.15 \pm 0.13$ & 2006 Feb 19 \\
\hline \multirow[t]{3}{*}{ 2MASS J04322946+1814002 } & $7.38 \pm 0.05$ & 2004 Feb 20 \\
\hline & $7.40 \pm 0.04$ & 2004 Sep 25 \\
\hline & $7.42 \pm 0.04$ & 2006 Feb 19 \\
\hline \multirow[t]{3}{*}{ IRAC J04323521+2420213 } & $7.06 \pm 0.05$ & 2004 Sep 25 \\
\hline & $7.07 \pm 0.05$ & 2005 Mar 1 \\
\hline & $7.10 \pm 0.05$ & 2007 Sep 23 \\
\hline \multirow[t]{4}{*}{ 2MASS J04325921+2430403 } & $8.30 \pm 0.09$ & 2004 Sep 25 \\
\hline & $8.49 \pm 0.16$ & 2005 Feb 26 \\
\hline & $8.81 \pm 0.22$ & 2005 Mar 2 \\
\hline & $8.59 \pm 0.07$ & 2007 Sep 24 \\
\hline 2MASS J04335562+2425016 & $8.40 \pm 0.08$ & 2005 Mar 1 \\
\hline 2MASS J04400363+2553547 & $7.33 \pm 0.05$ & 2005 Mar 4 \\
\hline \multirow[t]{4}{*}{ IRAC J04412575+2543492 } & $6.51 \pm 0.04$ & 2004 Sep 25 \\
\hline & $6.63 \pm 0.04$ & 2005 Feb 26 \\
\hline & $6.71 \pm 0.05$ & 2005 Feb 28 \\
\hline & $6.52 \pm 0.04$ & 2007 Sep 25 \\
\hline 2MASS J04455482+2408435 & $5.00 \pm 0.04$ & 2005 Feb 28 \\
\hline
\end{tabular}

Tau, we noticed a fainter object at a separation of 5 .'7, which we confirmed spectroscopically as a young brown dwarf. We discussed this pair in detail in a separate study (Luhman et al. 2009b).

By inspecting images of the remaining new members, we have identified two additional candidate companions. The new member IRAS $04125+2902$ is $4^{\prime \prime}$ from a source that is $\sim 2 \mathrm{mag}$ fainter and is detected by DSS, 2MASS, and IRAC. This candidate, 2MASS J04154269+2909558, does not exhibit midIR excess emission in the IRAC data, indicating that it is a class III source if it is a member of Taurus. The second candidate companion, 2MASS J04355949+2238291, is $10^{\prime \prime}$ from XEST 09-042. Although it has red mid-IR colors, it is probably a galaxy rather than a young star based on the shape of its SED.

\section{INITIAL MASS FUNCTION}

We wish to use our updated census of Taurus to estimate the IMF in this region. The completeness of the current census is a function of several parameters, including stellar mass, location, SED class, and extinction. Therefore, we must carefully define the sample of members for inclusion in the IMF so that it is representative of the stellar population in Taurus. We begin by considering members of Taurus that are located within the areas observed by XEST, which are shown in Figure 1. We select the XEST fields because they encompass a large number of members and are almost entirely covered by deep imaging at optical and IR wavelengths as well as in X-rays (Briceño et al. 2002; Luhman 2004c, 2006; Luhman et al. 2006; Guieu et al. 2006). To evaluate the completeness of the census of Taurus within these fields, we first examine the completeness of XEST. In Figure 11, we plot the distributions of spectral types for all known members of Taurus within the XEST images and for the members detected in those data. Separate histograms are shown for classes I, II, and III, where the SED classifications are from Luhman et al. (2009a). We omit secondaries that are unresolved by XEST. An additional 16 members are absent from Figure 11 because they lack accurate spectral types, five of which were detected by XEST. All of these unclassified stars have class I 


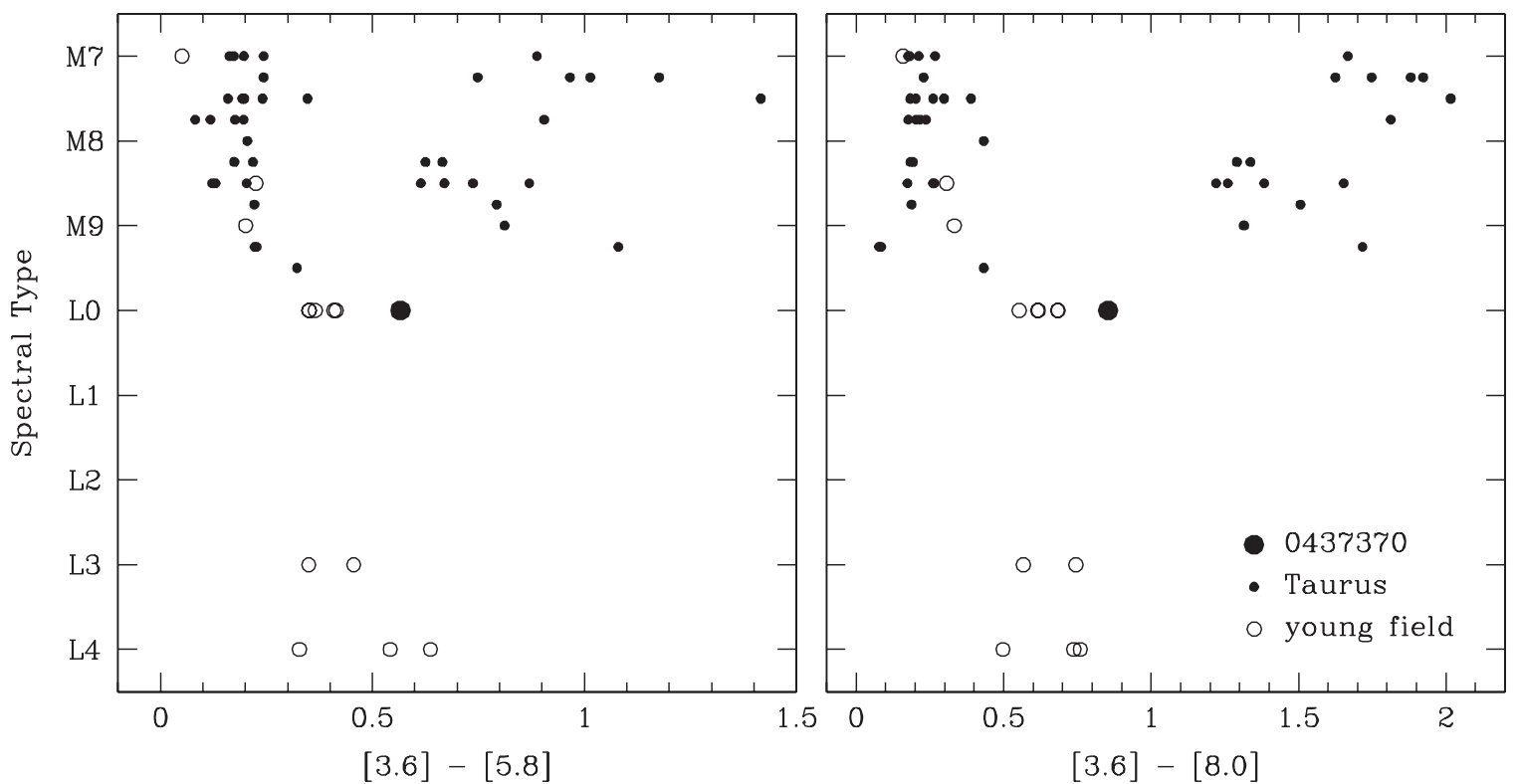

Figure 8. Spectral types vs. mid-IR colors for late-type members of Taurus (filled circles, $\tau \sim 1 \mathrm{Myr}$ ) and young field dwarfs (open circles, $\tau \lesssim 100 \mathrm{Myr}$ ). The blue sequence in each color represents stellar photospheres while the redder objects are likely to have circumstellar disks. The new L0 member of Taurus (large filled circle) does not exhibit significant color excesses relative to young L-type members of the field (Kirkpatrick et al. 2006, 2008; Cruz et al. 2009).

Table 7

IRAC Photometry for Young Cool Field Dwarfs

\begin{tabular}{|c|c|c|c|c|c|c|}
\hline ID & $\begin{array}{c}\text { Spectral } \\
\text { Type }^{\mathrm{a}} \\
\end{array}$ & [3.6] & {$[4.5]$} & {$[5.8]$} & {$[8.0]$} & Date \\
\hline 2MASS J00332386-1521309 & L4 & $12.54 \pm 0.02$ & $12.48 \pm 0.02$ & $12.21 \pm 0.03$ & $12.04 \pm 0.03$ & 2007 Aug 10 \\
\hline 2MASS J01415823-4633574 & L0 & $12.36 \pm 0.02$ & $12.17 \pm 0.02$ & $11.95 \pm 0.03$ & $11.68 \pm 0.03$ & 2006 Aug 12 \\
\hline 2MASS J02411151-0326587 & L0 & $13.39 \pm 0.02$ & $13.24 \pm 0.02$ & $13.04 \pm 0.03$ & $12.77 \pm 0.03$ & 2007 Sep 12 \\
\hline 2MASSI J0253597+320637 & M7 & $12.17 \pm 0.02$ & $12.12 \pm 0.02$ & $12.12 \pm 0.03$ & $12.01 \pm 0.03$ & 2007 Sep 7 \\
\hline 2MASS J03231002-4631237 & L0 & $12.84 \pm 0.02$ & $12.68 \pm 0.02$ & $12.48 \pm 0.03$ & $12.16 \pm 0.03$ & 2007 Aug 10 \\
\hline 2MASS J03572695-4417305 & L0 & $12.21 \pm 0.02$ & $12.08 \pm 0.02$ & $11.86 \pm 0.03$ & $11.65 \pm 0.03$ & 2007 Aug 10 \\
\hline SDSS J044337.61+000205.1 & M9 & $10.55 \pm 0.02$ & $10.45 \pm 0.02$ & $10.35 \pm 0.03$ & $10.22 \pm 0.03$ & 2007 Oct 16 \\
\hline 2MASS J05012406-0010452 & $\mathrm{L} 4$ & $11.77 \pm 0.02$ & $11.52 \pm 0.02$ & $11.22 \pm 0.03$ & $11.03 \pm 0.03$ & 2008 Mar 9 \\
\hline 2MASSI J0608528-275358 & M8.5 & $11.75 \pm 0.02$ & $11.62 \pm 0.02$ & $11.52 \pm 0.03$ & $11.44 \pm 0.03$ & 2007 Oct 16 \\
\hline 2MASSI J1615425+495321 & $\mathrm{L} 4$ & $12.91 \pm 0.02$ & $12.61 \pm 0.02$ & $12.28 \pm 0.03$ & $12.15 \pm 0.03$ & $2007 \mathrm{Jul} 2$ \\
\hline 2MASSI J1726000+153819 & L3 & $12.76 \pm 0.02$ & $12.64 \pm 0.02$ & $12.41 \pm 0.03$ & $12.20 \pm 0.03$ & 2007 Sep 7 \\
\hline 2MASSW J2208136+292121 & L3 & $13.08 \pm 0.02$ & $12.89 \pm 0.02$ & $12.62 \pm 0.03$ & $12.33 \pm 0.03$ & 2007 Jul 3 \\
\hline 2MASS J22134491-2136079 & L0 & $12.99 \pm 0.02$ & $12.83 \pm 0.02$ & $12.58 \pm 0.03$ & $12.37 \pm 0.03$ & 2007 Jun 29 \\
\hline
\end{tabular}

Note. ${ }^{a}$ Kirkpatrick et al. (2006, 2008) and Cruz et al. (2007, 2009).

SEDs. Only 15 of the 31 class I sources within the XEST fields have spectral classifications and thus are present in Figure 11. According to Figure 11, the completeness of XEST decreases for lower stellar masses and earlier SED classes, which is a reflection of the fact that X-ray emission is correlated with both of these properties (Telleschi et al. 2007; Prisinzano et al. 2008). XEST is nearly $100 \%$ complete for class III members earlier than M6 $\left(M \sim 0.1 M_{\odot}\right)$. Grosso et al. (2007) reported that XEST detected eight of 16 members later than M6 that are within those images. Using our updated census of Taurus and the spectral types that we have measured in this work and in previous studies, we find that the XEST detection fraction is 7/17 for types later than M6.

Optical and IR surveys for new members of Taurus have complemented XEST in terms of completeness. Images from Spitzer have covered $\sim 92 \%$ of the XEST fields (Luhman et al. 2009a). The current census for that portion of the XEST fields is nearly complete for class I and II stars and for class II brown dwarfs down to $\sim 0.02 M_{\odot}$ according to Spitzer surveys for new members (Luhman et al. 2006; this work). The completeness for class I brown dwarfs is unknown because of contamination of the Spitzer images by red galaxies (Luhman et al. 2006). The remaining $\sim 8 \%$ of the XEST fields that lack Spitzer images does not encompass any known members or dark clouds, and thus probably does not contain a significant number of undiscovered class I and II sources. Meanwhile, the XEST fields have been fully covered at optical and near-IR wavelengths by either deep wide-field imaging (Briceño et al. 2002; Luhman 2004c; Guieu et al. 2006) or all-sky catalogs (Luhman 2006), which have produced a high level of completeness for classes II and III between 0.1 and $0.02 M_{\odot}$ within an extinction range that encompasses most members $\left(A_{V}<4\right.$; Luhman 2004c, 2006; Guieu et al. 2006). Thus, the X-ray, optical, and IR studies have produced a census of Taurus members within the areas covered by XEST that should be complete down to masses of $\sim 0.02 M_{\odot}$ for classes II and III.

As done for the H-R diagram in Section 4.1, we treat multiple systems that are unresolved by 2MASS as single sources when 


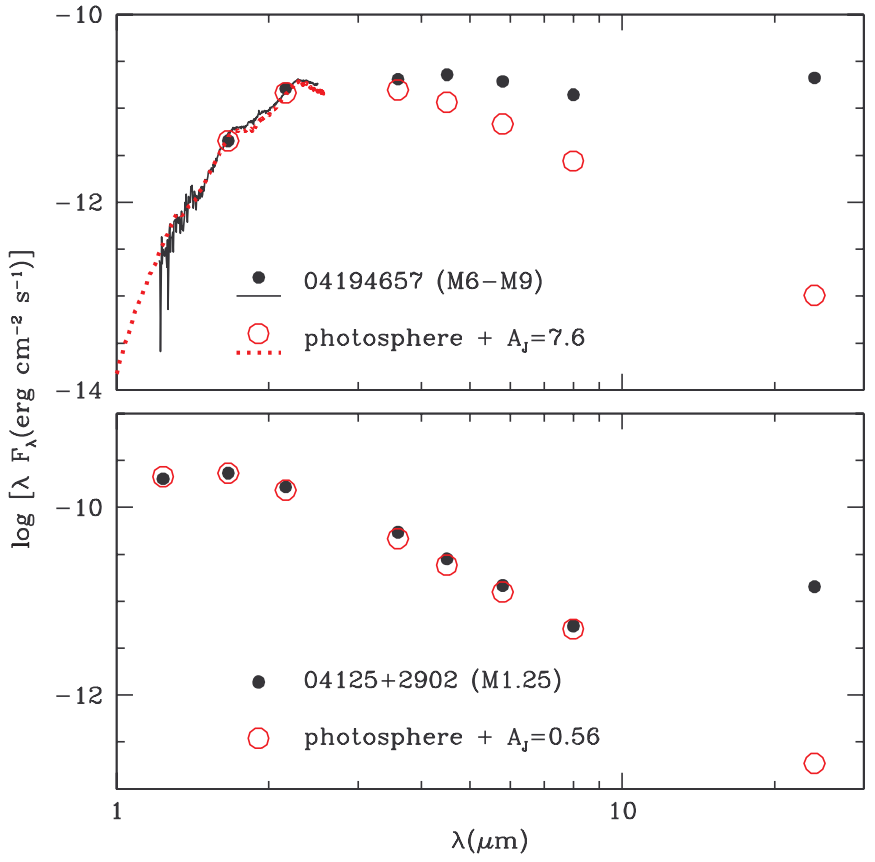

Figure 9. Two notable SEDs among the new members of Taurus (filled circles and solid line) compared to SEDs of young stellar photospheres with similar spectral types (open circles and dotted line). 2MASS J04194657+2712552 has the highest extinction of any known late-type member of Taurus and exhibits a flat mid-IR SED, suggesting that it may have a protostellar envelope. IRAS $04125+2902$ shows excess emission at $24 \mu \mathrm{m}$ but not at $\lambda \leqslant 8 \mu \mathrm{m}$, which is a signature of a disk with an inner hole. Each photospheric SED has been reddened by the extinction of the Taurus source and scaled to its $H$-band flux.
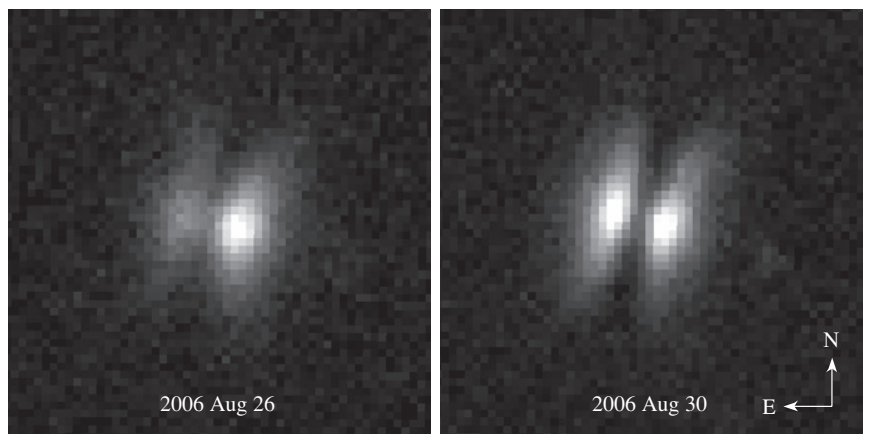

Figure 10. I-band CFHT images of the new Taurus member 2MASS J04202144+2813491 $\left(10^{\prime \prime} \times 10^{\prime \prime}\right)$. These data exhibit bipolar extended emission separated by a dark lane, indicating the presence of an edge-on circumstellar disk that is occulting the star. The brightness of the eastern lobe of scattered light varied significantly between these two dates. Point sources in the surrounding areas of these images exhibit FWHM $=0.17$ (left) and 0.'5 (right).

constructing the IMF for the XEST fields. We exclude class I sources, stars that lack accurate spectral classifications (most of which are class I), and objects that are probably seen in scattered light based on their unusually low luminosity estimates. The resulting mass function consists of primaries and widely separated secondaries, as in our previous measurements of IMFs in Taurus and other star-forming regions (Luhman et al. 2003b; Luhman 2004c, 2007). We have estimated the masses for the Taurus members in the IMF sample from their positions on the H-R diagram in Figure 7 by using the theoretical evolutionary models of Baraffe et al. (1998) and Chabrier et al. (2000) for $M / M_{\odot} \leqslant 1$ and the models of Palla \& Stahler (1999) for $M / M_{\odot}>1$. The IMF for the XEST fields is presented in Figure 12 in logarithmic units where the Salpeter
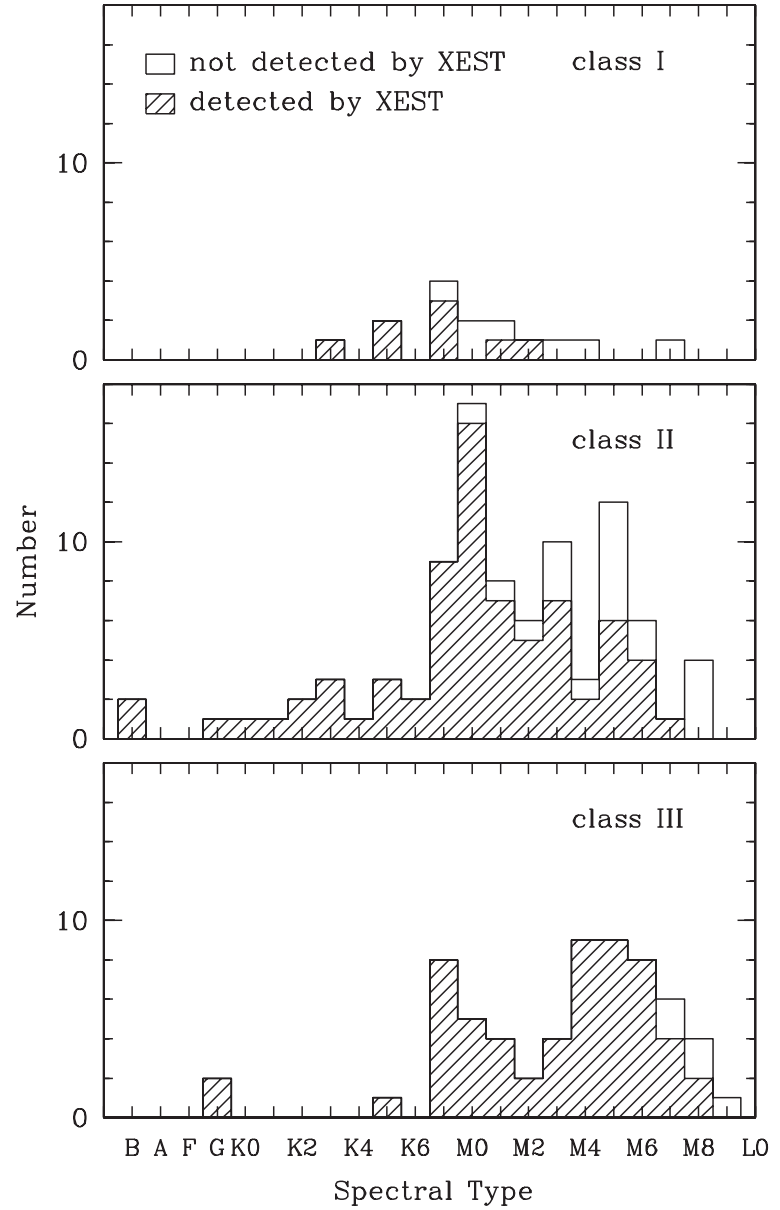

Figure 11. Distributions of spectral types for known members of Taurus that are within the XEST fields as a function of SED class. The stars that were detected by XMM-Newton are indicated (shaded histograms).

slope is $\alpha=1.35$. It exhibits a maximum near $0.8 M_{\odot}$ and declines steadily to lower masses $(\alpha=-0.44)$, and thus closely resembles the IMFs that we have previously reported for Taurus (Briceño et al. 2002; Luhman 2004c). In comparison, the mass functions of other nearby star-forming regions peak at $0.1-$ $0.2 M_{\odot}$ (Hillenbrand 1997; Hillenbrand \& Carpenter 2000; Muench et al. 2002, 2003; Luhman et al. 2003b; Luhman 2007). This variation in the IMF is illustrated in Figure 12, where we include mass functions for IC 348 and Chamaeleon I that were derived in the same manner as our measurement for Taurus (Luhman et al. 2003b; Luhman 2007). The surplus of stars near $0.8 M_{\odot}(\mathrm{K} 7-\mathrm{M} 1)$ in Taurus relative to those two clusters is also apparent in the distributions of spectral types for the IMF samples in Figure 13. Based on a two-sided Kolmogorov-Smirnov test, the probability that the sample for Taurus was drawn from the same mass distribution as either IC 348 or Chamaeleon I is $\sim 0.04 \%$. Possible explanations for the distinctive shape of the IMF in Taurus have been discussed in previous studies and generally involve a higher average Jeans mass in this region (Briceño et al. 2002; Luhman et al. 2003b; Luhman 2004c; Goodwin et al. 2004; Lada et al. 2008).

Finally, we comment briefly on another young population in which an unusual IMF has been reported. By constructing an IMF from all known members of the $\eta$ Cha association (Mamajek et al. 1999; Lawson et al. 2002; Lyo et al. 2004; Song et al. 2004; Luhman \& Steeghs 2004), Lyo et al. (2004, 2006) found that $\eta$ Cha exhibits a deficit of 20-29 low-mass stars and brown dwarfs $\left(0.025-0.15 M_{\odot}\right)$ relative to the solar 

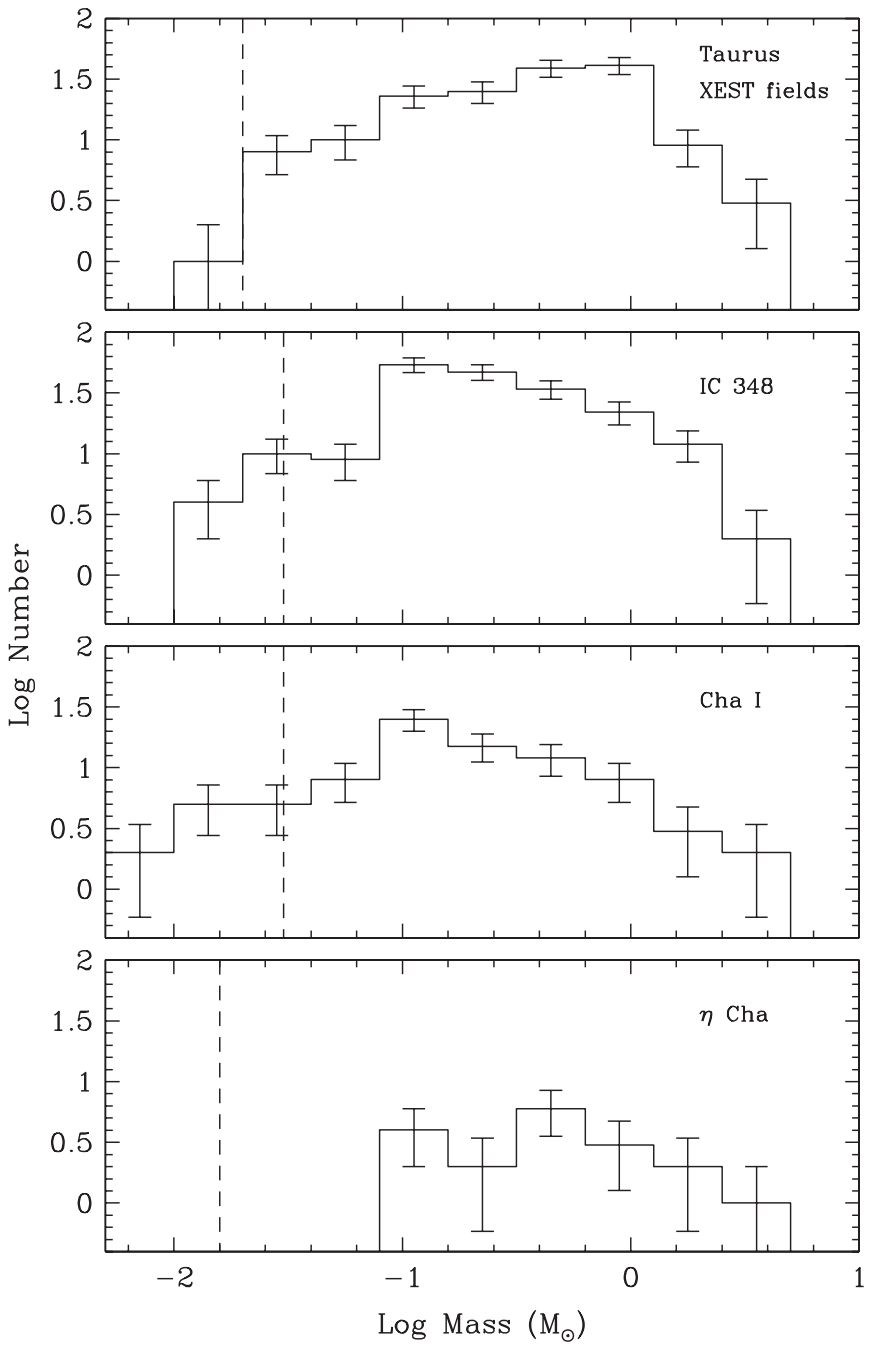

Figure 12. IMFs for Taurus, IC 348 (Luhman et al. 2003b), and Chamaeleon I (Luhman 2007). The IMF for Taurus is derived from members within the XEST fields (Figure 1). These IMFs contain 157, 194, and 85 sources, respectively. The Taurus IMF differs significantly from the mass functions in the other two regions (Section 5). We also include the IMF of the $\eta$ Cha association (Mamajek et al. 1999; Lawson et al. 2002; Lyo et al. 2004; Song et al. 2004; Luhman \& Steeghs 2004). Although Lyo et al. (2004) reported that $\eta$ Cha exhibits a deficit of low-mass stars and brown dwarfs relative to other clusters, its mass function is statistically consistent with the IMFs of IC 348 and Chamaeleon I (Luhman 2004b; Section 5). The completeness limits of these samples are indicated (dashed lines). In the units of this diagram, the Salpeter slope is 1.35 .

neighborhood and other young clusters. Moraux et al. (2007) attempted to provide a theoretical explanation for the apparently unusual IMF in this association. However, through a survey of $\eta$ Cha that was complete to $0.015 M_{\odot},{ }^{7}$ Luhman \& Steeghs (2004) and Luhman (2004b) concluded that a significant paucity of lowmass objects is not present. To further explore this issue, we have constructed an IMF and a distribution of spectral types for all known members of $\eta$ Cha, which are included in Figures 12 and 13, respectively. A two-sided Kolmogorov-Smirnov test

\footnotetext{
7 Lyo et al. (2006) and Moraux et al. (2007) incorrectly quoted a completeness limit of $0.025 M_{\odot}$ for the surveys by Luhman \& Steeghs (2004) and Luhman (2004b), which employed photometry from DENIS and 2MASS. Lyo et al. (2006) suggested that the completeness limit from those surveys was determined by their shallowest data, which consisted of the optical photometry from DENIS. However, any objects above $0.015 M_{\odot}$ that were absent from the optical data would have appeared in the IR diagrams used by Luhman \& Steeghs (2004) and Luhman (2004b) for selecting candidate members. Thus, the completeness limit was $0.015 M_{\odot}$.
}
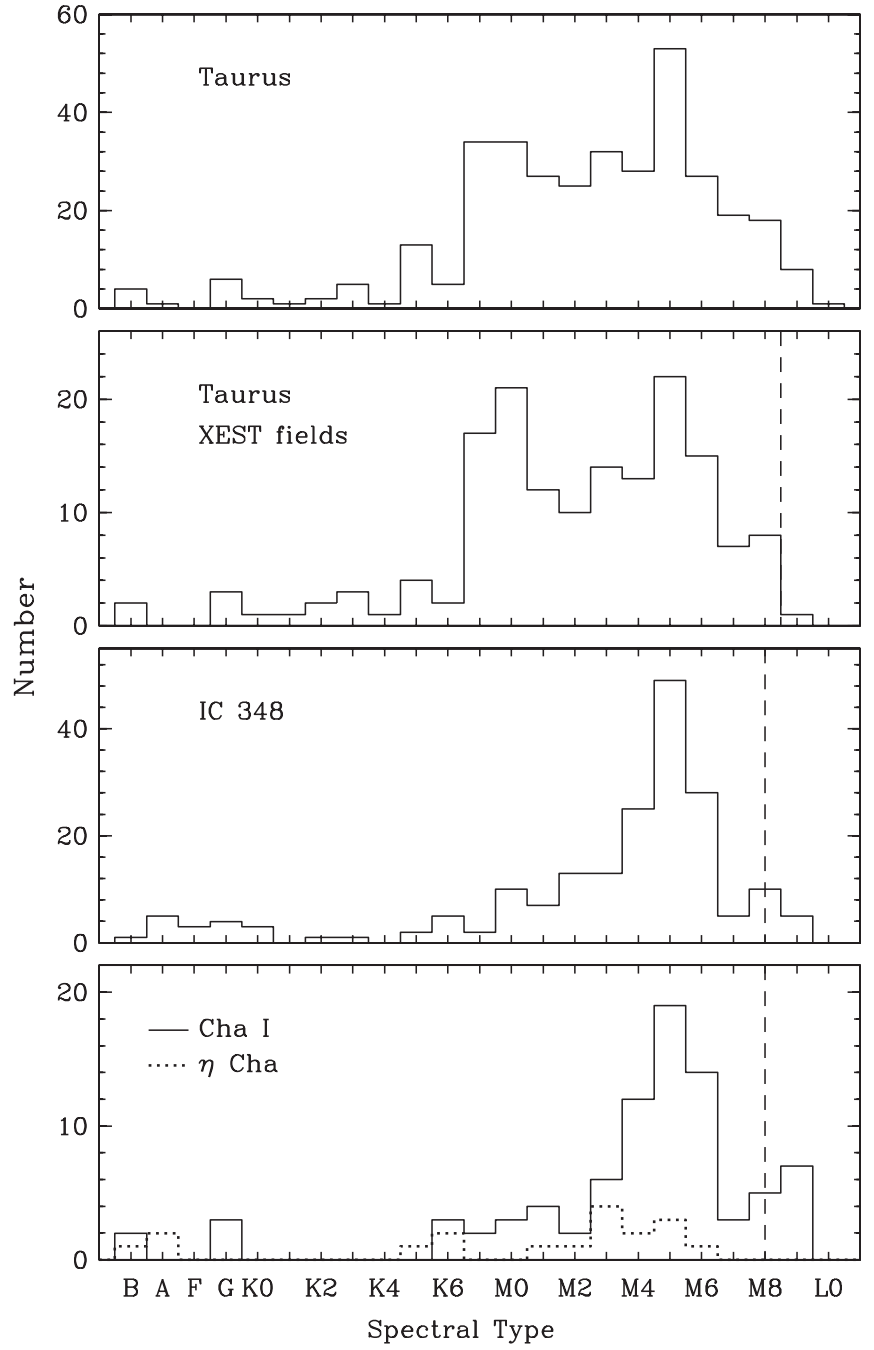

Figure 13. Distributions of spectral types for all known members of Taurus and for the IMFs of Taurus, IC 348, Chamaeleon I, and $\eta$ Cha in Figure 12. The completeness limits of the IMF samples in Taurus, IC 348, and Chamaeleon I are indicated (dashed lines). The limit for $\eta$ Cha is near M9.

indicates a probability of $\sim 10 \%$ that the members of $\eta$ Cha are drawn from the same mass distribution as either IC 348 or Chamaeleon I, which does not represent a significant difference. Thus, the IMF in $\eta$ Cha is consistent with the mass functions in IC 348 and Chamaeleon I.

\section{CONCLUSIONS}

We have presented a survey for new members of the Taurus star-forming region in which we obtained spectra of candidate members appearing in images from the Spitzer Space Telescope $\left(46 \mathrm{deg}^{2}\right)$ and the XMM-Newton Observatory $\left(5 \mathrm{deg}^{2}\right)$. Using the mid-IR data from Spitzer, we identified 44 sources that could be young stars with disks, 24 of which were confirmed as members by our spectroscopy. We also performed spectroscopy on 51 candidates detected in X-rays by the XEST program (Güdel et al. 2007; Scelsi et al. 2007), demonstrating the youth and membership of 16 sources. Ten of these new X-ray members were independently confirmed through spectroscopy by Scelsi et al. (2008). In addition to the sources from Spitzer and XMMNewton, we observed four candidate companions to known members of Taurus that were found by Kraus \& Hillenbrand (2007) through analysis of 2MASS data, one of which we have classified as a young star. 


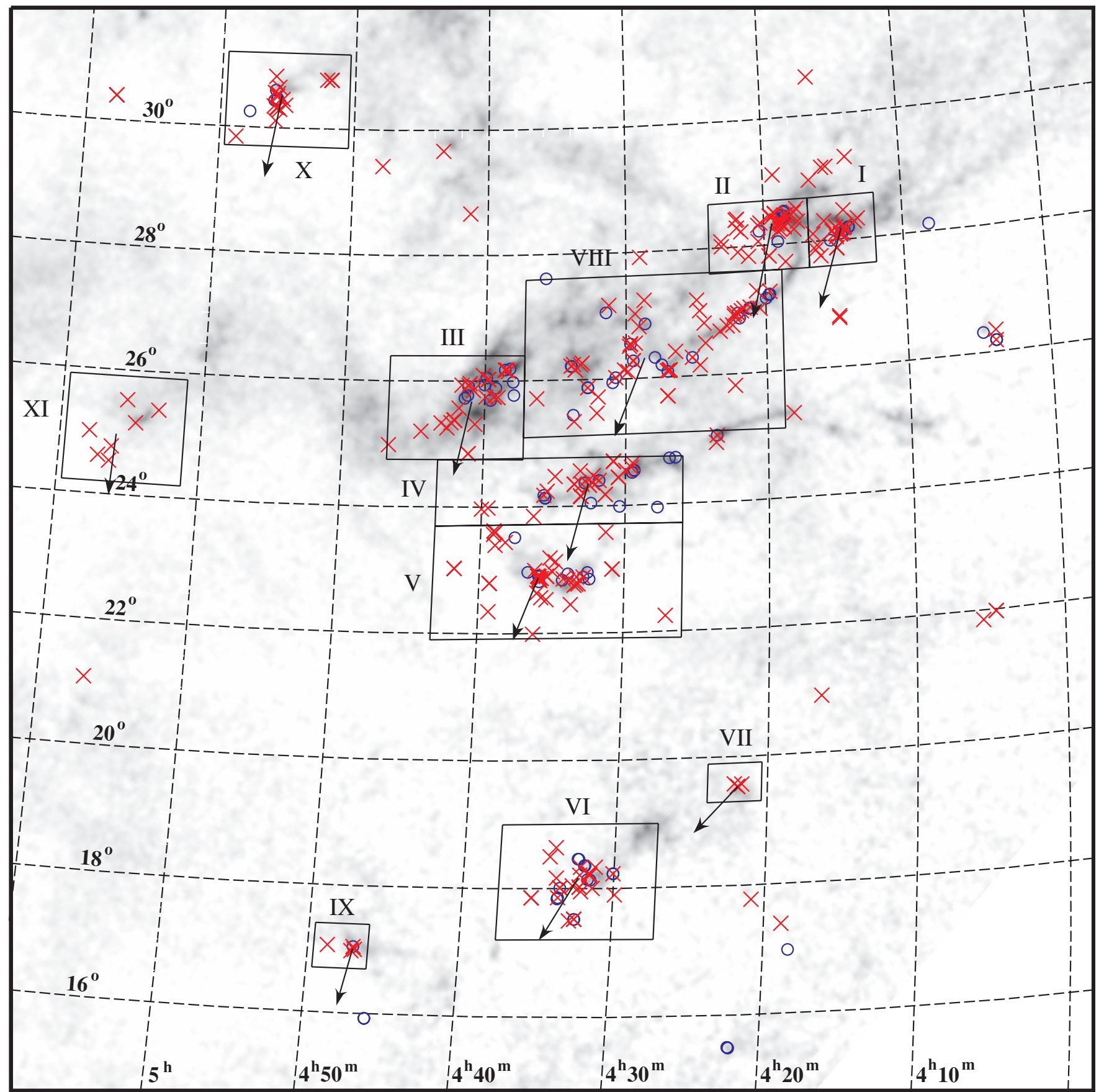

Figure 14. Spatial distribution of all known members of the Taurus star-forming region, which are labeled according to the presence or absence of proper motion measurements (crosses and circles). The median proper motions of members within 11 groups (rectangles) are presented in Table 8. The corresponding motions over a period of $0.2 \mathrm{Myr}$ are indicated (arrows). The dark clouds in Taurus are displayed with a map of extinction (gray scale, Dobashi et al. 2005).

Our survey has uncovered several rare types of sources that are valuable for studies of various aspects of star and planet formation. They consist of a wide binary brown dwarf that is forming in isolation (Luhman et al. 2009b), the first known L-type member of Taurus $\left(M \sim 4-7 M_{\text {Jup }}\right)$, a highly reddened brown dwarf that may be in the class I stage (star+disk+envelope), a disk that appears to have an inner hole (i.e., transitional disk), and a large edge-on disk $(r=2.5=350 \mathrm{AU})$. The companion identified by Kraus \& Hillenbrand (2007) also may comprise the primary in wide, low-mass binary system (M4.5+M8.5, $a=12^{\prime \prime}=$ 1700 AU). Meanwhile, the Spitzer and XMM-Newton data in conjunction with previous optical and near-IR surveys provide relatively well defined completeness limits for the current census of Taurus, enabling a better characterization of the stellar population. For instance, we have estimated the IMF within the fields observed by XEST, arriving at a distribution that reaches a maximum near $0.8 M_{\odot}$, which agrees with our previous measurements for Taurus. Thus, the IMF in Taurus continues to appear anomalous compared to other nearby star-forming clusters, which peak at $0.1-0.2 M_{\odot}$. The disk fraction in the XEST fields and the spatial distribution of the SED classes are investigated by Luhman et al. (2009a).

The completeness of the census of Taurus remains poorly determined among class I sources at low masses and class III 
Table 8

Median Positions, Proper Motions, and Velocities of Taurus Groups

\begin{tabular}{|c|c|c|c|c|c|c|c|c|c|c|c|}
\hline Group $^{\mathrm{a}}$ & $\alpha(\mathrm{J} 2000)$ & $\delta(\mathrm{J} 2000)$ & $\mu_{\alpha}^{\mathrm{b}}$ & $\mu_{\delta}^{\mathrm{b}}$ & $\mathrm{N}_{\mu}^{\mathrm{c}}$ & $\mathrm{RV}_{\text {star }}{ }^{\mathrm{d}}$ & $\mathrm{RV}_{\text {gas }}{ }^{\mathrm{d}}$ & $U^{\mathrm{e}}$ & $V^{\mathrm{e}}$ & $W^{\mathrm{e}}$ & Cloud \\
\hline I & 41426 & 281200 & +6.9 & -22.3 & 63 & $12.3 \pm 2.5$ & 15.5 & -15 & -11 & -11 & B209 \\
\hline II & 41924 & 282020 & +6.0 & -26.8 & 101 & $14.9 \pm 0.6$ & 15.6 & -15 & -13 & -13 & L1495E \\
\hline III & 44055 & 254050 & +4.5 & -21.3 & 57 & $16.3 \pm 1.0$ & 15.9 & -15 & -11 & -10 & L1527 \\
\hline IV & 43236 & 242140 & +5.5 & -21.9 & 79 & $16.8 \pm 0.7$ & 16.6 & -15 & -12 & -11 & L1529 \\
\hline $\mathrm{V}$ & 43555 & 225250 & +6.7 & -17.7 & 88 & $15.7 \pm 1.5$ & 16.4 & -15 & -11 & -9 & L1536 \\
\hline VI & 43222 & 181050 & +10.0 & -17.6 & 87 & $17.9 \pm 0.3$ & 18.5 & -17 & -13 & -8 & L1551 \\
\hline VII & 42158 & 193220 & +12.2 & -12.7 & 12 & $17.6 \pm 1.0$ & $\ldots$ & -17 & -11 & -6 & NGC1554 \\
\hline VIII & 42841 & 261910 & +8.6 & -22.0 & 111 & $14.9 \pm 0.6$ & 16.4 & -16 & -13 & -10 & B217 \\
\hline IX & 44700 & 170040 & +3.2 & -16.1 & 12 & $22.5 \pm 1.1$ & 20.6 & -18 & -11 & -11 & L1558 \\
\hline$X$ & 45529 & 303040 & +3.9 & -23.4 & 52 & $14.8 \pm 0.5$ & 14.9 & -15 & -12 & -10 & L1517 \\
\hline XI & 50600 & 245810 & +0.9 & -17.6 & 24 & $16.8 \pm 3.8$ & 17.9 & -17 & -9 & -9 & L1544 \\
\hline All of Taurus & 43210 & 255140 & +6.1 & -21.0 & 757 & $16.3 \pm 0.3$ & 16.4 & -16 & -11 & -10 & $\ldots$ \\
\hline
\end{tabular}

Notes. Proper motions $\left(\mu_{\alpha}, \mu_{\delta}\right)$ and velocities $(\mathrm{RV}, U, V, W)$ have units of mas $\mathrm{yr}^{-1}$ and $\mathrm{km} \mathrm{s}^{-1}$, respectively.

${ }^{a}$ Group names I through VI are from Gomez et al. (1993). We have defined the designations for the remaining groups.

${ }^{\mathrm{b}}$ Uncertainties in the median $\mu_{\alpha}$ and $\mu_{\delta}$ values for each group are $\sim 1 \mathrm{mas} \mathrm{yr}^{-1}$ for groups I, II, IV, VI, VIII, X, and XI, and $\sim 2$ mas yr ${ }^{-1}$ for groups III, V, VII, and IX.

${ }^{\mathrm{c}}$ Number of proper motion measurements included in the calculation of the median motion.

d Heliocentric velocities.

e Galactic Cartesian velocities.

sources outside of the XEST fields, which focused on the denser stellar aggregates. Future surveys can address these shortcomings through spectroscopy of red, faint sources detected by Spitzer and measurements of variability and proper motions with wide-field, multi-epoch imaging (e.g., Panoramic Survey Telescope and Rapid Response System, Large Synoptic Survey Telescope).

K. L. was supported by grant AST-0544588 from the National Science Foundation. This work makes use of data from the Spitzer Space Telescope and 2MASS. Spitzer is operated by the Jet Propulsion Laboratory, California Institute of Technology under a contract with NASA. 2MASS is a joint project of the University of Massachusetts and the Infrared Processing and Analysis Center/California Institute of Technology, funded by NASA and the NSF. Support for K. C. was provided by NASA through the Spitzer Space Telescope Fellowship Program through a contract issued by JPL/Caltech. XMM-Newton is an ESA science mission with instruments and contributions directly funded by ESA Member States and NASA. The HET is a joint project of the University of Texas at Austin, the Pennsylvania State University, Stanford University, Ludwig-MaximilliansUniversität München, and Georg-August-Universität Göttingen. The HET is named in honor of its principal benefactors, William P. Hobby and Robert E. Eberly. The Marcario LowResolution Spectrograph at HET is named for Mike Marcario of High Lonesome Optics, who fabricated several optics for the instrument but died before its completion; it is a joint project of the HET partnership and the Instituto de Astronomía de la Universidad Nacional Autónoma de México. MegaPrime/ MegaCam is a joint project of CFHT and CEA/DAPNIA. CFHT is operated by the National Research Council of Canada, the Institute National des Sciences de l'Univers of the Centre National de la Recherche Scientifique of France, and the University of Hawaii. Gemini Observatory is operated by AURA under a cooperative agreement with the NSF on behalf of the Gemini partnership: the NSF (United States), the Particle Physics and Astronomy Research Council (United Kingdom), the National Research Council (Canada), CONICYT (Chile), the Australian Research Council (Australia), CNPq (Brazil) and
CONICET (Argentina). This research has made use of data obtained from the SuperCOSMOS Science Archive, prepared and hosted by the Wide Field Astronomy Unit, Institute for Astronomy, University of Edinburgh, which is funded by the UK Science and Technology Facilities Council.

\section{APPENDIX}

\section{ADOPTED CENSUS OF TAURUS}

Our analysis of the IMF in Taurus required a census of all known members of the region. While constructing this census, we have made use of proper motion measurements when they are available. For a star whose membership is uncertain, we have compared its proper motion to that of the nearest group of known members. In Table 8, we present the median proper motions of 11 groups in Taurus based on stars from our adopted census and proper motions measurements from a variety of sources (Harris et al. 1999; Høg et al. 2000; Hambly et al. 2001; Hanson et al. 2004; Zacharias et al. 2004a, 2004b; Ducourant et al. 2005; Loinard et al. 2007; van Leeuwen 2007; Röser et al. 2008; Torres et al. 2007, 2009). Our adopted boundaries for these groups and the median motions are shown on a map of Taurus in Figure 14.

We describe our adopted census of known members of Taurus in terms of modifications to the compilation of members presented by Kenyon et al. (2008) in a review of this star-forming region. We begin by identifying the sources from that list that we have excluded as members. The proper motions of HBC

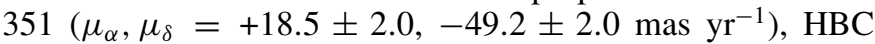
$352\left(+6 \pm 1,-9 \pm 1\right.$ mas $\left.\mathrm{yr}^{-1}\right), \mathrm{HBC} 353(+7 \pm 8,-12 \pm$

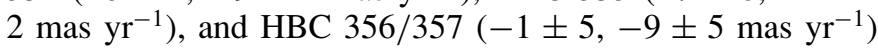
from Zacharias et al. (2004a, 2004b) differ significantly from those of the nearest Taurus groups in Table 8. The available proper motion measurements for HBC 354 and HBC 355, which are separated by $6^{\prime \prime}$, also are inconsistent with membership in Taurus (Zacharias et al. 2004a; Ducourant et al. 2005; Röser et al. 2008). We provided some of the new members reported in this work to Kenyon et al. (2008) for inclusion in their list of members, including 2MASS J04345973+2807017 and 2MASS J04124858+2749563. However, upon closer examination of these two stars, we have concluded that they are probably not 
members of Taurus (Section 3.2). Our spectroscopy indicates that IRAS $04428+2403$ is a galaxy. No evidence of membership has been presented for V410 Anon 20 and V410 Anon 24. They are fainter than other Taurus members near their spectral types, suggesting that they are background stars. CIDA-13 and St34 have been classified as probable foreground stars (Muzerolle et al. 2003; Hartmann et al. 2005b).

In addition to the members compiled by Kenyon et al. (2008), we have included in our census the new members presented in Table 2 as well as 2MASS J04162725+2053091, 2MASS J04270739+2215037, 2MASS J04344544+2308027, 2MASS J04381630+2326402, 2MASS J04385859+2336351, 2MASS J04385871+2323595, 2MASS J04390163+2336029, 2MASS J04390637+2334179 (Slesnick et al. 2006), 2MASS J04295422+1754041, 2MASS J04263055+2443558 (Luhman 2006), IRAS 04325+2402C (Hartmann et al. 1999), IRAS 04111+2800G (Prusti et al. 1992), L1521F-IRS (Bourke et al. 2006), IRAM 04191+1522 (André et al. 1999), IRAS 04278+2253 B (White \& Hillenbrand 2004), LH 0429+17 (Harris et al. 1999; Reid \& Hawley 1999), and HD 28867 (Walter et al. 2003). 2MASS J04333278+1800436 was identified as a possible member of Taurus by Walter et al. (2003) based on its close proximity to HD 28867 . We add it to our census since it exhibits mid-IR excess emission that indicates the presence of a disk (Luhman et al. 2009a). The proper motions of HD $30171\left(+12.1 \pm 1.1,-17.7 \pm 1.1 \mathrm{mas} \mathrm{yr}^{-1}\right.$, Høg et al. 2000) and MWC $480\left(+5.5 \pm 1.1,-25.4 \pm 1.1 \mathrm{mas} \mathrm{yr}^{-1}, \mathrm{Høg}\right.$ et al. 2000) are similar to those of the nearest Taurus groups (Table 8), supporting their membership in Taurus.

We have found a few other differences between our census and that of Kenyon et al. (2008). The coordinates that we retrieved from the 2MASS Point Source Catalog differ from those in Kenyon et al. (2008) by $2^{\prime \prime}-22^{\prime \prime}$ for IRAS 04016+2610, HBC 412, IRAS 04370+2559, MHO 9, CIDA12, and IRAS 04191+1523. Our adopted 2MASS counterparts for these stars are given in Luhman et al. (2009a). IRAS 04181+2655 appears in the census from Kenyon et al. (2008), but it is a blend of three other stars in that list, 2MASS J04210795+2702204, 2MASS J04211038+2701372, and 2MASS J04211146+2701094, and thus does not require a separate entry. Similarly, IRAS $04263+2426$ and GV Tau are listed separately by Kenyon et al. (2008) even though they represent the same star. The coordinates for IRAS 04166+2706 in Kenyon et al. (2008) apply to IRAS $04166+2708$. We have measured coordinates of $\alpha=4^{\mathrm{h}} 19^{\mathrm{m}} 42^{\mathrm{s}} .5, \delta=27^{\circ} 13^{\prime} 36^{\prime \prime} .7(\mathrm{~J} 2000)$ for the true counterpart to IRAS $04166+2706$ using images from Spitzer. The misidentification for IRAS 04166+2706 in Kenyon et al. (2008) probably originated in Luhman (2006) and Luhman et al. (2006). Our adopted list of Taurus members is provided in Luhman et al. (2009a).

By combining our membership list with previous astrometric and kinematic measurements for these stars, we can constrain both the radial velocities and three-dimensional velocities of the Taurus groups in Table 8 . For each group, we have calculated the median radial velocity of stars (Appenzeller et al. 1988; Barbier-Brossat et al. 1994; Barbier-Brossat \& Figon 2000; Finkenzeller \& Jankovics 1984; Gahm et al. 1999; Hartmann et al. 1986, 1987; Herbig 1977; Malaroda et al. 2006; Martín et al. 2005; Mathieu et al. 1997; Mundt et al. 1983; Reipurth et al. 1990; Sartoretti et al. 1998; Walter 1986; Walter et al. 1988; White \& Basri 2003; Zaitseva et al. 1985, 1990) and the median radial velocity of the dense gas based on observations of $\mathrm{C}^{18} \mathrm{O}$, $\mathrm{CS}, \mathrm{N}_{2} \mathrm{H}^{+}$, and $\mathrm{H}^{13} \mathrm{CO}^{+}$(Lee et al. 1999; Onishi et al. 2002;
Tatematsu et al. 2004). The gas radial velocity for group IX is from Ungerechts \& Thaddeus (1987). The gas velocities were transformed to the heliocentric frame using the standard solar motion (Kerr \& Lynden-Bell 1986). The median values of the stellar and gas radial velocities differ by an average of $-0.5 \pm$ $0.3 \mathrm{~km} \mathrm{~s}^{-1}\left(\mathrm{RV}_{\text {star }}-\mathrm{RV}_{\text {gas }}\right)$ and agree within $2.5 \sigma$ for all groups, providing further evidence that the stars and gas in Taurus share similar motions (e.g., Herbig 1977; Hartmann et al. 1986). Using the median positions, proper motions, and gas radial velocities (except for group VII, where $\mathrm{RV}_{\text {star }}$ is adopted), we have calculated velocities of the Taurus groups in the directions of the Galactic center $(U)$, Galactic rotation $(V)$, and the north Galactic pole $(W)$. Since accurate distances are available only for a few individual stars (Torres et al. 2009), we have adopted a distance of $140 \pm 10 \mathrm{pc}$ for each group. The uncertainties in each velocity component are $\pm 0.9-1.5 \mathrm{~km} \mathrm{~s}^{-1}$ with a mean error $1.0 \mathrm{~km} \mathrm{~s}^{-1}$. We derive a mean velocity for the Taurus complex of $U, V, W=-15.7 \pm 0.7,-11.3 \pm 0.7,-10.1 \pm 0.7 \mathrm{~km} \mathrm{~s}^{-1}$, which agrees with the mean value from Bertout et al. (2007). However, the one-dimensional velocity dispersions among the groups $\left(\sim 1 \mathrm{~km} \mathrm{~s}^{-1}\right)$ are much smaller than those estimated by Bertout et al. $\left(2007 ; \sim 6 \mathrm{~km} \mathrm{~s}^{-1}\right)$, who included a significant population of off-cloud sources. The groups appear to have rather coherent motions, suggesting that many of the off-cloud sources are unrelated to the Taurus dark clouds.

\section{REFERENCES}

Allen, L. E., \& Strom, K. M. 1995, AJ, 109, 1379

André, P., Motte, F., \& Bacmann, A. 1999, ApJ, 513, L57

Appenzeller, I., Reitermann, A., \& Stahl, O. 1988, PASP, 100, 815

Baraffe, I., Chabrier, G., Allard, F., \& Hauschildt, P. H. 1998, A\&A, 337, 403

Barbier-Brossat, M., \& Figon, P. 2000, A\&AS, 142, 217

Barbier-Brossat, M., Petit, M., \& Figon, P. 1994, A\&AS, 108, 603

Bertout, C., Siess, L., \& Cabrit, S. 2007, A\&A, 473, L21

Bourke, T. L., et al. 2006, ApJ, 649, L37

Briceño, C., Luhman, K. L., Hartmann, L., Stauffer, J. R., \& Kirkpatrick, J. D. 2002, ApJ, 580, 317

Brown, J. M., Blake, G. A., Qi, C., Dullemond, C. P., \& Wilner, D. J. 2008, ApJ, 675, L109

Burrows, C. J., et al. 1996, ApJ, 473, 437

Calvet, N., D’Alessio, P., Hartmann, L., Wilner, D., Walsh, A., \& Sitko, M. 2002, ApJ, 568, 1008

Calvet, N., et al. 2005, ApJ, 630, L185

Chabrier, G., Baraffe, I., Allard, F., \& Hauschildt, P. 2000, ApJ, 542, L119

Cruz, K. L., Kirkpatrick, J. D., \& Burgasser, A. J. 2009, AJ, 137, 3345

Cruz, K. L., et al. 2007, AJ, 133, 439

Cushing, M. C., Rayner, J. T., \& Vacca, W. D. 2005, ApJ, 623, 1115

Cushing, M. C., Vacca, W. D., \& Rayner, J. T. 2004, PASP, 116, 362

D’Alessio, P., et al. 2005, ApJ, 621, 461

Dobashi, K., Uehara, H., Kandori, R., Sakurai, T., Kaiden, M., Umemoto, T., \& Sato, F. 2005, PASJ, 57, 1

Ducourant, C., Teixeira, R., Périé, J. P., Lecampion, J. F., Guibert, J., \& Sartori, M. J. 2005, A\&A, 438, 769

Dutrey, A., et al. 2008, A\&A, 490, L15

Espaillat, C., et al. 2007a, ApJ, 664, L111

Espaillat, C., et al. 2007b, ApJ, 670, L135

Faherty, J. K., Burgasser, A. J., Cruz, K. L., Shara, M. M., Walter, F. M., \& Gelino, C. R. 2009, AJ, 137, 1

Fazio, G. G., et al. 2004, ApJS, 154, 10

Finkenzeller, U., \& Jankovics, I. 1984, A\&AS, 57, 285

Flaherty, K. M., Pipher, J. L., Megeath, S. T., Winston, E. M., Gutermuth, R. A., Muzerolle, J., Allen, L. E., \& Fazio, G. G. 2007, ApJ, 663, 1069

Furlan, E., et al. 2006, ApJS, 165, 568

Furlan, E., et al. 2007, ApJ, 644, 1176 
Furlan, E., et al. 2008, ApJS, 176, 184

Gahm, G. F., Petrov, P. P., Duemmler, R., Gameiro, J. F., \& Lago, M. T. V. T. 1999, A\&A, 352, L95

Gomez, M., Hartmann, L., Kenyon, S. J., \& Hewett, R. 1993, AJ, 105, 1927

Goodwin, S. P., Whitworth, A. P., \& Ward-Thompson, D. 2004, A\&A, 419, 543

Grosso, N., et al. 2007, A\&A, 468, 391

Güdel, M., et al. 2007, A\&A, 468, 353

Guieu, S., Dougados, C., Monin, J.-L., Magnier, E., \& Martín, E. L. 2006, A\&A, 446,485

Guieu, S., et al. 2007, A\&A, 465, 855

Hambly, N. C., Davenhall, A. C., Irwin, M. J., \& MacGillivray, H. T. 2001, MNRAS, 326, 1315

Hanson, R. B., Klemola, A. R., Jones, B. F., \& Monet, D. G. 2004, AJ, 128, 1430

Harris, S., Clegg, P., \& Hughes, J. 1988, MNRAS, 235, 441

Harris, H. C., et al. 1999, AJ, 117, 339

Hartmann, L., Calvet, N., Allen, L., Chen, H., \& Jayawardhana, R. 1999, AJ, 118,1784

Hartmann, L., Hewett, R., Stahler, S., \& Mathieu, R. D. 1986, ApJ, 309, 275

Hartmann, L., Megeath, S. T., Allen, L., Luhman, K., Calvet, N., D’Alessio, P., Franco-Hernandez, R., \& Fazio, G. 2005a, ApJ, 629, 881

Hartmann, L., et al. 2005b, ApJ, 628, L147

Hartmann, L. W., Soderblom, D. R., \& Stauffer, J. R. 1987, AJ, 93, 907

Henry, T. J., Kirkpatrick, J. D., \& Simons, D. A. 1994, AJ, 108, 1437

Herbig, G. H. 1977, ApJ, 214, 747

Hillenbrand, L. A. 1997, AJ, 113, 1733

Hillenbrand, L. A., \& Carpenter, J. M. 2000, ApJ, 540, 236

Høg, E., et al. 2000, A\&A, 355, L27

Hughes, A. M., Wilner, D. J., Calvet, N., D’Alessio, P., Claussen, M. J., \& Hogerheijde, M. R. 2007, ApJ, 664, 536

Hughes, A. M., et al. 2009, ApJ, 698, 131

Jansen, F., et al. 2001, A\&A, 365, L1

Jones, B. F., \& Herbig, G. H. 1979, AJ, 84, 1872

Kenyon, S. J., Gómez, M., Marzke, R. O., \& Hartmann, L. 1994, AJ, 108, 251

Kenyon, S. J., Gómez, M., \& Whitney, B. A. 2008, in Handbook of Star Forming Regions, Vol. 1, The Northern Sky, ASP Monograph Series 4, ed. B. Reipurth (San Francisco, CA: ASP), 405

Kerr, F. J., \& Lynden-Bell, D. 1986, MNRAS, 221, 1023

Kirkpatrick, J. D., Henry, T. J., \& Irwin, M. J. 1997, AJ, 113, 1421

Kirkpatrick, J. D., Henry, T. J., \& McCarthy, D. W. 1991, ApJS, 77, 417

Kirkpatrick, J. D., et al. 1999, ApJ, 519, 802

Kirkpatrick, J. D., et al. 2006, ApJ, 639, 1120

Kirkpatrick, J. D., et al. 2008, ApJ, 689, 1295

Kraus, A. L., \& Hillenbrand, L. A. 2007, ApJ, 662, 413

Lada, C. J. 1987, in IAU Symp. 115, Star Forming Regions, ed. M. Peimbert \& J. Jugaku (Dordrecht: Reidel), 1

Lada, C. J., Muench, A. A., Rathborne, J., Alves, J. F., \& Lombardi, M. 2008, ApJ, 672, 410

Lawson, W. A., Crause, L. A., Mamajek, E. E., \& Feigelson, E. D. 2002, MNRAS, 329, L29

Lee, C. W., Myers, P. C., \& Tafalla, M. 1999, ApJ, 526, 788

Loinard, L., Mioduszewski, A. J., Rodríguez, L. F., González, R. A., Rodríguez, M. I., \& Torres, R. M. 2005, ApJ, 619, L179

Loinard, L., et al. 2007, ApJ, 671, 546

Luhman, K. L. 1999, ApJ, 525, 466

Luhman, K. L. 2004a, ApJ, 602, 816

Luhman, K. L. 2004b, ApJ, 616, 1033

Luhman, K. L. 2004c, ApJ, 617, 1216

Luhman, K. L. 2006, ApJ, 645, 676

Luhman, K. L. 2007, ApJS, 173, 104

Luhman, K. L., Allen, P. R., Espaillat, C., Hartmann, L., \& Calvet, N. 2009a, ApJS, submitted

Luhman, K. L., Briceño, C., Stauffer, J. R., Hartmann, L., Barrado y Navascués, D., \& Nelson, C. 2003a, ApJ, 590, 348

Luhman, K. L., Mamajek, E. E., Allen, P. R., Muench, A. A., \& Finkbeiner, D. P. 2009b, ApJ, 691, 1265

Luhman, K. L., \& Muench, A. A. 2008, ApJ, 684, 654

Luhman, K. L., Stauffer, J. R., Muench, A. A., Rieke, G. H., Lada, E. A., Bouvier, J., \& Lada, C. J. 2003b, ApJ, 593, 1093

Luhman, K. L., \& Steeghs, D. 2004, ApJ, 609, 917

Luhman, K. L., Whitney, B. A., Meade, M. R., Babler, B. L., Indebetouw, R., Bracker, S., \& Churchwell, E. B. 2006, ApJ, 647, 1180

Luhman, K. L., et al. 2008, ApJ, 675, 1375

Lyo, A.-R, Lawson, W. A., Feigelson, E. D., \& Crause, L. A. 2004, MNRAS, 347,246

Lyo, A.-R, Song, I., Lawson, W. A., Bessell, M. S., \& Zuckerman, B. 2006, MNRAS, 368, 1451
Malaroda, S., Levato, H., \& Galliani, S. 2006, VizieR Online Data Catalog, 3249, 0

Mamajek, E., Lawson, W. A., \& Feigelson, E. D. 1999, ApJ, 516, 77

Martín, E. L., Magazzù, A., Delfosse, X., \& Mathieu, R. D. 2005, A\&A, 429, 939

Mathieu, R. D., Stassun, K., Basri, G., Jensen, E. L. N., Johns-Krull, C. M., Valenti, J. A., \& Hartmann, L. W. 1997, AJ, 113, 1841

Mohanty, S., Jayawardhana, R., \& Basri, G. 2005, ApJ, 626, 498

Monet, D. G., et al. 2003, AJ, 125, 984

Moraux, E., Lawson, W. A., \& Clarke, C. 2007, A\&A, 473, 163

Muench, A. A., Lada, C. J., Luhman, K. L., Muzerolle, J., \& Young, E. 2007, AJ, 134,411

Muench, A. A., Lada, E. A., Lada, C. J., \& Alves, J. 2002, ApJ, 573, 366

Muench, A. A., et al. 2003, AJ, 125, 2029

Mundt, R., Walter, F. M., Feigelson, E. D., Finkenzeller, U., Herbig, G. H., \& Odell, A. P. 1983, ApJ, 269, 229

Muzerolle, J., Hillenbrand, L., Calvet, N., Briceño, C., \& Hartmann, L. 2003, ApJ, 592, 266

Muzerolle, J., Luhman, K. L., Briceño, C., Hartmann, L., \& Calvet, N. 2005, ApJ, 625, 906

Onishi, T., Mizuno, A., Kawamura, A., Tachihara, K., \& Fukui, Y. 2002, ApJ, 575,950

Palla, F., \& Stahler, S. W. 1999, ApJ, 525, 772

Prisinzano, L., et al. 2008, ApJ, 677, 401

Prusti, T., Clark, F. O., Laureijs, R. J., Wakker, B. P., \& Wesselius, P. R. 1992, A\&A, 259, 537

Rayner, J. T., et al. 2003, PASP, 115, 362

Reid, I. N., \& Hawley, S. L. 1999, AJ, 117, 343

Reipurth, B., Lindgren, H., Nordstrom, B., \& Mayor, M. 1990, A\&A, 235, 197

Rieke, G. H., \& Lebofsky, M. J. 1985, ApJ, 288, 618

Rieke, G. H., et al. 2004, ApJS, 154, 25

Röser, S., Schilbach, E., Schwan, H., Kharchenko, N. V., Piskunov, A. E., \& Scholz, R.-D. 2008, A\&A, 488, 401

Sartoretti, P., Brown, R. A., Latham, D. W., \& Torres, G. 1998, A\&A, 334, 592

Scelsi, L., et al. 2007, A\&A, 468, 405

Scelsi, L., et al. 2008, A\&A, 490, 601

Schmidt-Kaler, T. 1982, in Landolt-Bornstein Group VI, Vol. 2, ed. K.-H. Hellwege (Berlin: Springer), 454

Skrutskie, M., et al. 2006, AJ, 131, 1163

Slesnick, C. L., Carpenter, J. M., Hillenbrand, L. A., \& Mamajek, E. E. 2006, AJ, 132,2665

Song, I., Zuckerman, B., \& Bessell, M. S. 2004, ApJ, 600, 1016

Tatematsu, K., Umemoto, T., Kandori, R., \& Sekimoto, Y. 2004, ApJ, 606, 333

Telleschi, A., Güdel, M., Briggs, K. R., Audard, M., \& Palla, F. 2007, A\&A, 468,425

Torres, C. A. O., Quast, G., de La Reza, R., Gregorio-Hetem, J., \& Lepine, J. R. D. 1995, AJ, 109, 2146

Torres, C. A. O., da Silva, L., Quast, G. R., de La Reza, R., \& Jilinksi, E. 2000, AJ, 120, 1410

Torres, R. M., Loinard, L., Mioduszewski, A. J., \& Rodríguez, L. F. 2007, ApJ, 671,1813

Torres, R. M., Loinard, L., Mioduszewski, A. J., \& Rodríguez, L. F. 2009, ApJ, 698,242

Ungerechts, H., \& Thaddeus, P. 1987, ApJS, 63, 645

Vacca, W. D., Cushing, M. C., \& Rayner, J. T. 2003, PASP, 115, 389

van Leeuwen, F. 2007, A\&A, 474, 653

Walter, F. M. 1986, ApJ, 306, 573

Walter, F. M., Beck, T. L., Morse, J. A., \& Wolk, S. J. 2003, AJ, 125, 2123

Walter, F. M., Brown, A., Mathieu, R. D., Myers, P. C., \& Vrba, F. J. 1988, AJ, 96, 297

Watson, A. M., \& Stapelfeldt, K. R. 2007, AJ, 133, 845

Werner, M. W., et al. 2004, ApJS, 154, 1

White, R. J., \& Basri, G. 2003, ApJ, 582, 1109

White, R. J., \& Ghez, A. M. 2001, ApJ, 556, 265

White, R. J., \& Hillenbrand, L. A. 2004, ApJ, 616, 998

Wichmann, R., Bastian, U., Krautter, J., Jankovics, I., \& Ruciński, S. M. 1998, MNRAS, 301, L39

Zacharias, N., Monet, D. G., Levine, S. E., Urban, S. E., Gaume, R., \& Wycoff, G. L. 2004a, BAAS, 36, 4815

Zacharias, N., Urban, S. E., Zacharias, M. I., Wycoff, G. L., Hall, D. M., Monet, D. G., \& Rafferty, T. J. 2004b, AJ, 127, 3043

Zaitseva, G. V., Kolotilov, E. A., Petrov, P. P., Tarasov, A. E., Shenavrin, V. I., \& Shcherbakov, A. G. 1985, Sov. Astron. Lett., 11, 109

Zaitseva, G. V., Shcherbakov, A. G., \& Stepanova, N. A. 1990, Sov. Astron. Lett., 16, 350

Zuckerman, B., \& Song, I. 2004, ARA\&A, 42, 685 